ESAIM: M2AN 48 (2014) 795-813

DOI: $10.1051 / \mathrm{m} 2 \mathrm{an} / 2013121$
ESAIM: Mathematical Modelling and Numerical Analysis

www.esaim-m2an.org

\title{
PARALLEL SCHWARZ WAVEFORM RELAXATION ALGORITHM FOR AN N-DIMENSIONAL SEMILINEAR HEAT EQUATION *
}

\author{
Minh-Binh Tran ${ }^{1}$
}

\begin{abstract}
We present in this paper a proof of well-posedness and convergence for the parallel Schwarz Waveform Relaxation Algorithm adapted to an N-dimensional semilinear heat equation. Since the equation we study is an evolution one, each subproblem at each step has its own local existence time, we then determine a common existence time for every problem in any subdomain at any step. We also introduce a new technique: Exponential Decay Error Estimates, to prove the convergence of the Schwarz Methods, with multisubdomains, and then apply it to our problem.
\end{abstract}

Mathematics Subject Classification. 65M12.

Received September 20, 2012. Revised August 12, 2013.

Published online April 1, 2014.

\section{INTRODUCTION}

In the pioneer work [16-18], P. L. Lions laid the foundations of the modern theory of Schwarz algorithms. He also proposed to use the Schwarz alternating method for evolution equations, and studied the algorithm for nonlinear monotone problems. Later, Schwarz waveform relaxation algorithms, by refering to the paper [2], were designed independently in $[12,14]$ for the linear advection-diffusion equation. They try to solve, on a given time interval, a sequence of Cauchy Problems with the transmission conditions of Cauchy type on overlapping subdomains. The algorithm is well-posed with some compatibility conditions.

An extension to the nonlinear reaction-diffusion equation in dimension 1 was considered in [10]. For nonlinear problems, especially evolutional equations, there are some cases that the solutions blow up in finite time, which means that if we divide the domain into several subdomains, at each step we can get different existence times in different domains, and we do not know if there exists a common existence time for all iterations. However, with the hypothesis $f^{\prime}(c) \leq C$ in [10], we do not encounter this difficulty and the iterations are defined naturally on an unbounded time interval. Proofs of linear convergence on unbounded time domains, and superlinear convergence on finite time intervals were then given in case of $n$ subdomains, based on some explicit computations on the linearized equations. Another extension to monotone nonlinear PDEs in higher dimensions were considered by Lui in $[19,20]$. In these papers, some monotone iterations for Schwarz methods are defined in order to get the convergence of the algorithm, based on the idea of the sub-super solutions method in PDEs and no explosion is considered. Recently, an extension to Systems of Semilinear Reaction-Diffusion Equations was investigated

Keywords and phrases. Domain decomposition, waveform relaxation, Schwarz methods, semilinear heat equation.

* The author has been partially supported by Grant MTM2011-29306-C02-00, MICINN, Spain, ERC Advanced Grant FPr246775 NUMERIWAVES, and Grant PI2010-04 of the Basque Government.

1 Basque Center for Applied Mathematics, Alameda de Mazarredo, 1448009 Bilbao, Basque Country, Spain. tbinh@bcamath. org 
in [5]. Some systems in dimension 1 were considered and the proofs of the well-posedness and the convergence of the algorithm used in this paper were a development of the technique used in [10].

We consider here the semilinear heat equation (2.1), in a spatial domain $\Omega=D \times(a, b)$ of $\mathbb{R}^{N}$, with the nonlinearity of the form (2.2), which allows explosion of solutions in finite time. We cut the domain into bands $\Omega_{i}=D \times\left(a_{i}, b_{i}\right)$, with $a_{1}=a$ and $b_{I}=b$. These bands are overlapping, i.e. for all $i \in\{1, I-1\}, a_{i+1}<b_{i}<b_{i+1}$. In each of these subdomains, we solve a heat equation with Dirichlet limit conditions. Since the domains are not smooth, we cannot use classical results about semilinear heat equations on smooth domains; we then establish some new proofs of existence for a general domain in Theorem 2.1. Applying the results in Theorem 2.1 for the equation (2.7), we get an existence result for a semilinear heat equation in a domain of the type $\Omega=D \times(a, b)$ in Theorem 2.2 .

Theorem 2.3 confirms that the algorithm is well-posed and there exists a common existence time for all subdomains at all iterations despite of the phenomenon of explosion. The common existence time $T^{*}$ is computed explicitly so that one can use it in numerical simulations. This is a collolary of Theorem 2.2.

We prove in Theorem 2.4 that the algorithm converges linearly. There are five main techniques to prove the convergence of Domain Decomposition Algorithms and they are: Orthorgonal Projection used for a linear Laplace equation (see [16]), Fourier and Laplace Transforms used for linear equations (see [8,9,11,14]), Maximum Priciple used for linear equations (see [13]), Energy Estimates used for nonoverlapping algorithms (see [1]), and Monotone Iterations (see [19,20]) used for nonlinear monotone problems. The convergence problem of overlapping algorithms for nonlinear equations is still open up to now. In this paper, we introduce a new technique: Exponential Decay Error Estimates, based on the idea of constructing some Controlling Functions, that allows us to prove that the algorithm converges linearly. An announcement of this research was published earlier in [22].

\section{The MAIN RESUlTS}

We consider the semilinear heat equation

$$
\partial_{t} u-\Delta u-f(u)=0
$$

with the assumptions on $f$ :

$$
f \text { is in } C^{1}(\mathbb{R}) \text { and there exists } C_{f}>0, p>1 \text { such that }\left|f^{\prime}(x)\right| \leq C_{f}|x|^{p-1}, \forall x \in \mathbb{R} .
$$

Remark 2.1. An example of this function is $f(x)=x^{p}$.

We first set an existence theorem for the initial boundary value problem, and more important, new estimates on the solution. We need here some notations. We set $p_{1}=\frac{3(p-1)}{4 p}, \alpha>0$ satisfying $1-\left(p_{1}+p \alpha\right)>0, l_{1}$ and $l_{2}$ are positive numbers such that $\frac{1}{l_{1}}+\frac{1}{l_{2}}=1$ and $l_{1} p_{1}<1$. We denote by $\|u\|_{k, h}$ the norm $\|u\|_{L^{k}\left(0, T, L^{h}(\omega)\right)}$, when $u$ belongs to $L^{k}\left(0, T, L^{h}(\omega)\right)$, where $\omega$ is some domain in $\mathbb{R}^{N}, k, h$ can be infinite. We define

$$
\begin{gathered}
\tau(r, m)=\left[(4 \pi)^{-p_{1}} \frac{2^{p_{1}+p \alpha}}{1-\left(p_{1}+p \alpha\right)} C_{f} \max \left(1,2^{p-2}\right)(4 r+4 m)\right]^{-\frac{8 p}{3+p}}, \\
G\left(r ; T, m_{1}, m_{2}\right)=\left(\frac{(4 \pi)^{-p_{1} l_{1}} T^{1-p_{1} l_{1}}}{1-p_{1} l_{1}}\right)^{-\frac{l_{2}}{l_{1}}} \int_{0}^{r}\left[C_{f} \max \left\{1,2^{p-2}\right\}\left(m_{1}+\zeta^{\frac{p-1}{l_{2}}}\right) \zeta^{\frac{1}{l_{2}}}+m_{2}\right]^{-l_{2}} \mathrm{~d} \zeta .
\end{gathered}
$$

Before studying (2.1), we will firstly consider the following problem

$$
\begin{cases}\partial_{t} w-\Delta w=f(w+v) & \text { in } \mathcal{O} \times(0, T), \\ w=0 & \text { on } \partial \mathcal{O} \times[0, T], \\ w(., 0)=0 & \text { in } \mathcal{O},\end{cases}
$$

where $\mathcal{O}$ is a bounded domain in $\mathbb{R}^{N}$. 
Theorem 2.2. Suppose that $\mathcal{O}$ is a bounded domain in $\mathbb{R}^{N}$ and denote its measure by $m(\mathcal{O})$.

Let $T_{0}$ be a positive constant and suppose that $v \in C\left(\left[0, T_{0}\right], L^{2}(\mathcal{O})\right)$ satisfying $|v| \leq M$ a.e.. Let $R_{1}=$ $2 \max _{|\zeta| \leq M}|f(\zeta)| m(\mathcal{O})^{\frac{1}{2}} \frac{T_{0}^{\frac{p+3}{4 p}}}{\frac{3(p-1)}{4 p}}$. Then, there exists a time $T_{*}=\min \left(T_{0}, 1, \tau\left(R_{1}, M^{p-1} m(\mathcal{O})^{\frac{p-1}{2 p}}\right)\right)$, such that for all $T<T_{*}$, equation (2.4) has a unique solution $w$ in $L^{\infty}(\mathcal{O} \times(0, T)) \cap C\left([0, T], L^{2}(\mathcal{O})\right) \cap L^{2}\left(0, T, H_{0}^{1}(\mathcal{O})\right)$ and $\partial_{t} w \in L^{2}\left(0, T, L^{2}(\mathcal{O})\right)$. Moreover, $\|w\|_{\infty, \infty} \leq M_{*}$, where

$$
\begin{gathered}
G^{-1}\left(T_{*}\right) \equiv G^{-1}\left(T_{*} ; T, M^{p-1} m(\mathcal{O})^{\frac{p-1}{2 p}}, m(\mathcal{O})^{\frac{1}{2}} \max _{|\zeta| \leq M}|f(\zeta)|\right) \\
M_{*}:=\left(\frac{4 T_{*}}{\pi^{3}}\right)^{\frac{1}{4}}\left[C_{f} \max \left(1,2^{p-2}\right)\left(G^{-1}\left(T_{*}\right)^{\frac{p-1}{l_{2}}}+M^{p-1} m(\mathcal{O})^{\frac{p-1}{2 p}}\right) G^{-1}\left(T_{*}\right)^{\frac{p-1}{l_{2}}}\right. \\
\left.+m(\mathcal{O})^{\frac{1}{2}} \max _{|\zeta| \leq M}|f(\zeta)|\right] .
\end{gathered}
$$

Let $T_{0}$ be a positive constant and suppose that $v \in C\left(\left[0, T_{0}\right], L^{2}(\mathcal{O})\right) \cap L^{\infty}\left(0, T_{0}, L^{2 p}(\mathcal{O})\right)$ a.e.. Denote $R_{2}=$ $8 p T_{0}^{\frac{p+3}{4 p}}\|f(v)\|_{\infty, 2} /(3(p-1))$. Then, there exists a time $T_{*}=\min \left(T_{0}, 1, \tau\left(R_{2},\|v\|_{L^{\infty}\left(0, T, L^{2 p}(\mathcal{O})\right.}^{p-1}\right)\right.$, such that for all $T<T_{*}$, equation (2.4) has a unique solution $w$ in $L^{\infty}(\mathcal{O} \times(0, T)) \cap C\left([0, T], L^{2}(\mathcal{O})\right) \cap L^{2}\left(0, T, H_{0}^{1}(\mathcal{O})\right)$ and $\partial_{t} w \in L^{2}\left(0, T, L^{2}(\mathcal{O})\right)$. Moreover, $\|w\|_{\infty, \infty} \leq M_{*}$, where

$$
\begin{aligned}
& G^{-1}\left(T_{*}\right) \equiv G^{-1}\left(T_{*} ; T,\|v\|_{\infty, 2 p}^{p-1},\|f(v)\|_{\infty, 2}\right) \\
& M_{*}:=\left(\frac{4 T_{*}}{\pi^{3}}\right)^{\frac{1}{4}}\left[C_{f} \max \left(1,2^{p-2}\right)\left(G^{-1}\left(T_{*}\right)^{\frac{p-1}{l_{2}}}+\|v\|_{\infty, 2 p}^{p-1}\right) G^{-1}\left(T_{*}\right)^{\frac{p-1}{l_{2}}}\right. \\
& \left.+\|f(v)\|_{\infty, 2}\right] \text {. }
\end{aligned}
$$

We consider now a bounded domain of the following form $\Omega=D \times(a, b) \subset \mathbb{R}^{N}$, where $D$ is a bounded domain with smooth enough boundary $\partial D$ in $\mathbb{R}^{N-2}$. The boundary $\partial \Omega$ of $\Omega$ is made of three parts, $\Gamma_{L}=\bar{D} \times\{a\}$, $\Gamma_{R}=\bar{D} \times\{b\}$, and $\Gamma_{C}=\partial D \times(a, b)$. Dirichlet data $g$ are given on the boundary $\partial \Omega \times(0, T)$, defined by $g_{L}$ on $\Gamma_{L}, g_{R}$ on $\Gamma_{R}, g_{C}$ on $\Gamma_{C}$. These functions are all continuous. We now introduce the basic initial boundary value problem for (2.4):

$$
\begin{array}{ll}
\partial_{t} u-\Delta u=f(u) & \text { in } \Omega \times(0, T), \\
u=g & \text { in } \partial \Omega \times(0, T), \\
u(., 0)=u_{0} & \text { in } \Omega .
\end{array}
$$

The following theorem will be proved later by using Theorem 2.2 .

Theorem 2.3. Suppose that $u_{0} \in C(\bar{\Omega})$ and $g \in C(\overline{\partial \Omega})$ with $\left.u_{0}\right|_{\partial \Omega}=g_{\mid t=0}$. Let $M$ be a positive constant satisfying $M>\max \left(\left\|u_{0}\right\|_{\infty},\|g\|_{\infty}\right)$. Suppose that $R_{1}$ is like in Theorem 2.2. There exists a limit time $T_{*}=$ $\min \left(1, \tau\left(R, M^{p-1} m(\Omega)^{\frac{p-1}{2 p}}\right)\right)$, such that for all $T<T_{*}$, equation $(2.7)$ has a solution $u$ in $L^{\infty}(\Omega \times(0, T)) \cap$ $C\left([0, T], L^{2}(\Omega)\right) \cap L^{2}\left(0, T, H_{0}^{1}(\Omega)\right) ; \partial_{t} u \in L^{2}\left(0, T, L^{2}(\Omega)\right)$. Moreover $\|u\|_{\infty, \infty} \leq M+M^{*}$ where $M^{*}$ is obtained from (2.5) by replacing $\mathcal{O}$ by $\Omega$ and $u$ is continuous on $\overline{\Omega \times(0, T)}$.

We divide the domain $\Omega$ into $I$ subdomains with $\Omega_{i}=D \times\left(a_{i}, b_{i}\right)$, with $a_{1}=a$ and $b_{I}=b$. We suppose that $a_{1}<a_{2}<b_{1}<b_{2}<\ldots<a_{I}<b_{I-1}<b_{I}$, we denote by $L_{i}$ the length of $\Omega_{i}: L_{i}=b_{i}-a_{i}$, and by $S_{i}$ the size of the overlap $S_{i}=b_{i}-a_{i+1}$.

The parallel Schwarz Waveform Relaxation Algorithm solves $I$ equations in $I$ subdomains instead of solving directly the main problem (2.7). The iterate $\# k$ in the $j$ th domain, denoted by $u_{j}^{k}$, is defined by

$$
\begin{cases}\partial_{t} u_{j}^{k}-\Delta u_{j}^{k}=f\left(u_{j}^{k}\right) & \text { in } \Omega_{j} \times(0, T), \\ u_{j}^{k}\left(\cdot, a_{j}, \cdot\right)=u_{j-1}^{k-1}\left(\cdot, a_{j}, \cdot\right) & \text { in } D \times(0, T), \\ u_{j}^{k}\left(\cdot, b_{j}, \cdot\right)=u_{j+1}^{k-1}\left(\cdot, b_{j}, \cdot\right) & \text { in } D \times(0, T) .\end{cases}
$$

Each iterate inherits the boundary conditions and the initial values of $u$ :

$$
u_{j}^{k}=g \text { on } \partial \Omega_{j} \cap \partial \Omega \times(0, T), \quad u_{j}^{k}(\cdot, \cdot, 0)=u_{0} \text { in } \Omega_{j},
$$




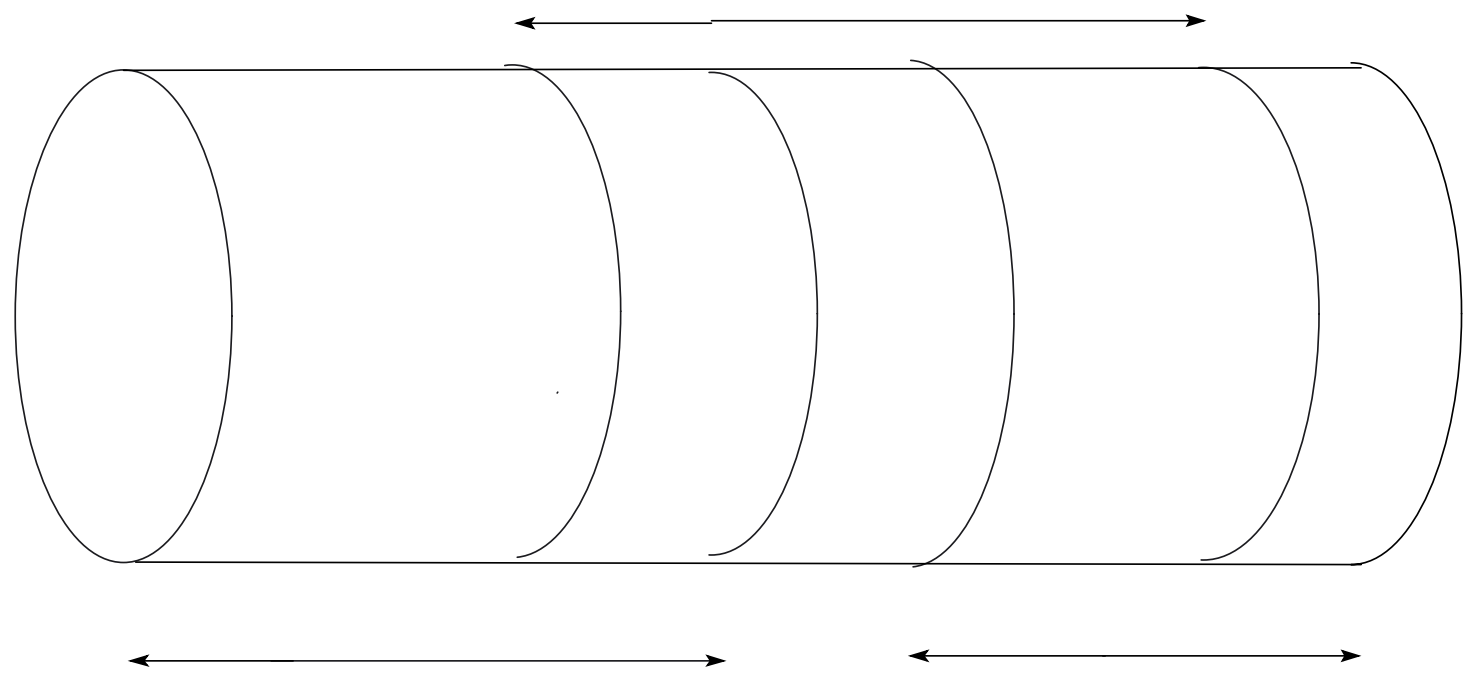

Figure 1. An illustration of how to divide $\Omega$.

which imposes a special treatment for the extreme subdomains,

$$
u_{1}^{k}(\cdot, a, \cdot)=g, \quad u_{I}^{k}(\cdot, b, \cdot)=g .
$$

An initial guess is provided, i.e. we solve at step 0 equations (2.8), with boundary data on left and right

$$
u_{j}^{0}\left(\cdot, a_{j}, \cdot\right)=g_{j}^{0} \text { in } D \times(0, T), u_{j}^{k}\left(\cdot, b_{j}, \cdot\right)=h_{j}^{0} \text { on } D \times(0, T) .
$$

Definition 2.4. The parallel Schwarz waveform relaxation algorithm is well-posed if there exists a local time $T^{*} \leq T_{*}$ such that for all $T<T^{*}$, each equation (2.8) in each iteration has a solution over the time interval $(0, T)$. Moreover, $\left\{u_{j}^{k}, j \in \overline{1, I}, k \in N\right\}$ is bounded in $C(\bar{\Omega} \times[0, T])$.

Let $M$ a positive number. According to theorem 2.3, the following problem has a solution $\phi_{M}$ in some interval $C\left(\bar{\Omega} \times\left[0, T_{0}\right]\right):$

$$
\begin{cases}\partial_{t} \phi_{M}-\Delta \phi_{M}=f\left(\phi_{M}\right) & \text { in } \Omega \times\left(0, T_{0}\right), \\ \phi_{M}=M & \text { in } \partial \Omega \times\left[0, T_{0}\right], \\ \phi_{M}(\cdot, \cdot, 0)=M & \text { in } \bar{\Omega} .\end{cases}
$$

The next theorem gives a common existence time for the iterates:

Theorem 2.5. Suppose the data $u_{0}$ and $g_{j}, g_{j}^{0}$ and $h_{j}^{0}$ are continuous and satisfy the compatibility conditions

$$
u_{0 \mid \partial \Omega}=g_{\mid t=0}, u_{0}\left(\cdot, a_{j}\right)=g_{j \mid t=0}^{0}, u_{0}\left(\cdot, b_{j}\right)=h_{j \mid t=0}^{0} .
$$

Let $M$ be a positive constant such that

$$
M>\max \left(\left\|u_{0}\right\|_{\infty},\|g\|_{\infty},\left(\left\|g_{i}^{0}\right\|_{\infty},\left\|h_{i}^{0}\right\|_{\infty}, i \in \overline{1, I}\right)\right) .
$$

Let $M_{*}$ be greater than the maximum of $\phi_{M}$ on the boundaries of $\Omega \times(0, T)$ and $\Omega_{j} \times(0, T), j \in \overline{1, I}$.

The algorithm (2.8) is then well-posed with a local time at least equal to

$$
\left.T^{*}=\min \left(T_{0}, 1, T_{*}, \tau\left(R_{1}, M_{*}^{p-1} m\left(\Omega_{j}\right)^{\frac{p-1}{2 p}}\right), j \in \overline{1, I}\right)\right) .
$$


We denote by $e_{j}^{k}$ the error $u_{j}^{k}-u$, where $u$ is the solution of (2.7). Let $P$ be a function from $\mathbb{R}$ to $\mathbb{R}$ such that (i) $P \in C^{2}(\mathbb{R})$; (i) $P(x), P^{\prime \prime}(x) \geq 0 \forall x \in \mathbb{R}$; (iii) $P(x)=0$ iff $x=0$ and $P^{\prime}(0)=0$; (iv) $\forall M>0$, there exists $K(M)$ such that $\left|\frac{x P^{\prime}(x)}{P(x)}\right|<K(M), \forall x \in \mathbb{R},|x|<M$. An example of $P$ is $P(x)=x^{2}$. We finally state the convergence of the algorithm:

Theorem 2.6. With the same assumptions as in Theorem 2.3, let $\gamma$ be a constant large enough and denote by $\bar{\epsilon}$ the constant $\sqrt{\frac{\gamma}{2}} \frac{S_{1} \ldots S_{I-1}}{L_{2} \ldots L_{I-1}}$. If we put $E_{k}=\max _{j \in \overline{1, I}}\left\|P\left(e_{j}^{k}\right) \exp (-\gamma t)\right\|_{C\left(\overline{\Omega_{j} \times(0, T)}\right)}=\max _{j \in \overline{1, I}} \| P\left(u_{j}^{k}-\right.$ u) $\exp (-\gamma t) \|_{C\left(\overline{\Omega_{j} \times(0, T)}\right)}$ and $\bar{E}_{k}=\max _{j \in \overline{1, I-1}}\left\{E_{k+j}\right\}$, the parallel Schwarz waveform relaxation algorithm (2.8) converges linearly in the following sense: for every $T<T^{*}$,

$$
\bar{E}_{n} \leq \bar{E}_{0} \exp (-n \bar{\epsilon}), \forall n \in \mathbb{N},
$$

and as a consequence

$$
\lim _{k \rightarrow \infty} \max _{j \in[1, I]}\left\|u_{j}^{k}-u\right\|_{C\left(\overline{\Omega_{j}} \times[0, T]\right)}=0 .
$$

\section{Proof of the EXISTENCE RESUlts For SEMILINEAR HeAT EQUATion IN AN ARBITRARY BOUNDED DOMAIN - THEOREM 2.2}

\subsection{Premiliary results}

Let $\omega$ be an arbitrary bounded domain in $\mathbb{R}^{N}$ and consider the following equation

$$
\begin{cases}\partial_{t} \zeta-\Delta \zeta=0 & \text { in } \omega \times(0, T), \\ \zeta(., .)=0 & \text { on } \partial \omega \times[0, T], \\ \zeta(., 0)=\zeta_{0} & \text { on } \bar{\omega} .\end{cases}
$$

We consider the operator $A \zeta=-\Delta \zeta, D(A)=\left\{\zeta \mid \zeta \in H_{0}^{1}(\omega), \Delta \zeta \in L^{2}(\omega)\right\}$. According to Proposition 2.6.1 page 26, of [3], $A$ is an m-dissipative operator with dense domain in $L^{2}(\omega)$. Let $S(t)$ be the Dirichlet semigroup associated with $A$ on $L^{2}(\omega)$ (see [4]). If $\zeta_{0} \in D(A)$, due to Theorem 3.1.1 page 33, of [3], $\zeta(t)=S(t) \zeta_{0}$ is a solution of (3.1).

According to Section 6.5 page 334, of [6], there exist a sequence of eigenvalues $0<\lambda_{1} \leq \lambda_{2} \ldots$ and $\lambda_{k} \rightarrow \infty$ as $k \rightarrow \infty$ and an othornormal basis $\left\{w_{k}\right\}_{k=1}^{\infty}$ of $L^{2}(\omega)$ which is also an orthogornal basis of $H_{0}^{1}(\omega)$, where $w_{k}$ is the eigenfunction corressponding to $\lambda_{k}$ :

$$
\begin{cases}A w_{k}=\lambda_{k} w_{k} & \text { in } \omega, \\ w_{k}=0 & \text { on } \partial \omega .\end{cases}
$$

Denote by $<,>$ the scalar product in $L^{2}(\omega)$, we have the following Lemmas, whose proofs are classical.

Lemma 3.1. If $\zeta_{0} \in L^{2}(\omega)$, then we have that

$$
S(t) \zeta_{0}=\sum_{k=1}^{\infty} \mathrm{e}^{-t \lambda_{k}}\left\langle\zeta_{0}, w_{k}\right\rangle w_{k}
$$

Lemma 3.2. Suppose that $g \in C\left([0, T], L^{2}(\omega)\right)$ and $\rho_{0} \in D(A)$, we consider the following equation

$$
\begin{cases}\partial_{t} \rho-\Delta \rho=g & \text { in } \omega \times(0, T), \\ \rho(., .)=0 & \text { on } \partial \omega \times[0, T], \\ \rho(., 0)=\rho_{0} & \text { on } \bar{\omega} .\end{cases}
$$


Then (3.3) has a solution in $L^{2}\left(0, T, H_{0}^{1}(\omega)\right) \cap L^{\infty}\left(0, T, L^{2}(\omega)\right)$ given by the following formula

$$
\rho(., t)=S(t) \rho_{0}+\int_{0}^{t} S(t-s) g(., s) \mathrm{d} s .
$$

\subsection{Proof of Theorem 2.2}

We consider again the operator $A \zeta=-\Delta \zeta, D(A)=\left\{\zeta \mid \zeta \in H_{0}^{1}(\mathcal{O}), \Delta \zeta \in L^{2}(\mathcal{O})\right\}$ with its associated Dirichlet semigroup $S(t)$. Thanks to Lemma 3.2, instead of considering directly the equation (2.4), in some of the following steps, we will consider the following equation

$$
u(t)=S(t)(0)+\int_{0}^{t} f(u+v)(s) \mathrm{d} s=\int_{0}^{t} f(u+v)(s) \mathrm{d} s .
$$

Step 1. We use a fixed point argument to prove that (3.5) has a solution.

We work with the case $v \in L^{\infty}\left(0, T, L^{2 p}(\mathcal{O})\right)$. The case $v \in L^{\infty}\left(0, T, L^{\infty}(\mathcal{O})\right)$ can be treated with exactly the same manner.

We consider the Banach space $Y_{T}=\left\{u \in L_{l o c}^{\infty}\left((0, T), L^{2 p}(\mathcal{O})\right),\|u\|_{Y_{T}}<\infty\right\}$ and $\|u\|_{Y_{T}}=$ $\sup _{0<t<T} t^{\alpha}\|u(., t)\|_{2 p}$. Let $B$ be the closed ball in $Y_{T}$ with center 0 and radius $R_{2}$, we will use the Banach Fixed Point Theorem for the mapping

$$
\begin{gathered}
\Phi: B \rightarrow B \\
\Phi(u)(., t)=\int_{0}^{t} S(t-s) f(u+v)(., s) \mathrm{d} s .
\end{gathered}
$$

Let $u_{1}, u_{2}$ be two functions in $B$, we now prove that $\Phi$ is a contraction

$$
t^{\alpha}\left\|\Phi\left(u_{1}\right)(., t)-\Phi\left(u_{2}\right)(., t)\right\|_{2 p} \leq t^{\alpha} \int_{0}^{t}\left\|S(t-s)\left(f\left(u_{1}+v\right)-f\left(u_{2}+v\right)\right)\right\|_{2 p} \mathrm{~d} s .
$$

Using the $L^{p}-L^{q}$ estimate (see [21] Prop. 48.4, p. 441), we obtain

$$
\left\|S(t-s)\left(f\left(u_{1}+v\right)(., s)-f\left(u_{2}+v\right)(., s)\right)\right\|_{2 p} \leq(4 \pi(t-s))^{-\frac{3(p-1)}{4 p}}\left\|f\left(u_{1}+v\right)(., s)-f\left(u_{2}+v\right)(., s)\right\|_{2} .
$$

This leads to

$$
\begin{aligned}
& t^{\alpha} \int_{0}^{t}\left\|S(t-s)\left(f\left(u_{1}+v\right)-f\left(u_{2}+v\right)\right)\right\|_{2 p} \mathrm{~d} s \\
\leq & t^{\alpha}(4 \pi)^{-\frac{3(p-1)}{4 p}} \int_{0}^{t}(t-s)^{-\frac{3(p-1)}{4 p}}\left\|f^{\prime}(v+\zeta)\right\|_{\frac{2 p}{p-1}}\left\|u_{1}-u_{2}\right\|_{2 p} \mathrm{~d} s \\
\leq & t^{\alpha}(4 \pi)^{-\frac{3(p-1)}{4 p}} \int_{0}^{t}(t-s)^{-\frac{3(p-1)}{4 p}}\left\|C_{f}\left|u_{1}+v\right|^{p-1}+C_{f}\left|u_{2}+v\right|^{p-1}||_{\frac{2 p}{p-1}}\right\| u_{1}-u_{2} \|_{2 p} \mathrm{~d} s \\
\leq & t^{\alpha}(4 \pi)^{-\frac{3(p-1)}{4 p}} C_{f} \int_{0}^{t}(t-s)^{-\frac{3(p-1)}{4 p}}\left(\left\|u_{1}+v\right\|_{2 p}^{p-1}+\left\|u_{2}+v\right\|_{2 p}^{p-1}\right)\left\|u_{1}-u_{2}\right\|_{2 p} \mathrm{~d} s .
\end{aligned}
$$

We have $\left\|u_{i}+v\right\|_{2 p}^{p-1} \leq\left(\left\|u_{i}\right\|_{2 p}+\|v\|_{2 p}\right)^{p-1}$, for $i=1,2$. Moreover, if $p \geq 2$, we have that $\left(\left\|u_{i}\right\|_{2 p}+\|v\|_{2 p}\right)^{p-1} \leq$ $2^{p-2}\left(\left\|u_{i}\right\|_{2 p}^{p-1}+\|v\|_{2 p}^{p-1}\right)$ and if $p<2$, we have that $\left(\left\|u_{i}\right\|_{2 p}+\|v\|_{2 p}\right)^{p-1} \leq\left\|u_{i}\right\|_{2 p}^{p-1}+\|v\|_{2 p}^{p-1}$, for $i=1$, 2. These 
inequalities lead to

$$
\begin{aligned}
& t^{\alpha} \int_{0}^{t}\left\|S(t-s)\left(f\left(u_{1}+v\right)(., s)-f\left(u_{2}+v\right)(., s)\right)\right\|_{2 p} \mathrm{~d} s \\
\leq & t^{\alpha}(4 \pi)^{-\frac{3(p-1)}{4 p}} C_{f} \max \left\{2^{p-2}, 1\right\} \\
& \times \int_{0}^{t}(t-s)^{-\frac{3(p-1)}{4 p}}\left(\left\|u_{1}\right\|_{2 p}^{p-1}+\left\|u_{2}\right\|_{2 p}^{p-1}+2\|v\|_{2 p}^{p-1}\right)\left\|u_{1}-u_{2}\right\|_{2 p} \mathrm{~d} s \\
\leq & t^{\alpha}(4 \pi)^{-\frac{3(p-1)}{4 p}} C_{f} \max \left\{2^{p-2}, 1\right\} \int_{0}^{t}(t-s)^{-\frac{3(p-1)}{4 p}} s^{-(p-1) \alpha} \\
& \times\left(s^{(p-1) \alpha}\left\|u_{1}\right\|_{2 p}^{p-1}+s^{(p-1) \alpha}\left\|u_{2}\right\|_{2 p}^{p-1}+2 s^{(p-1) \alpha}\|v\|_{2 p}^{p-1}\right)\left\|u_{1}-u_{2}\right\|_{2 p} \mathrm{~d} s \\
\leq & t^{\alpha}(4 \pi)^{-\frac{3(p-1)}{4 p}} C_{f} \max \left\{2^{p-2}, 1\right\} \\
& \times \int_{0}^{t}(t-s)^{-\frac{3(p-1)}{4 p}} s^{-(p-1) \alpha}\left(2 R_{2}+2 T^{(p-1) \alpha}\|v\|_{2 p}^{p-1}\right)\left\|u_{1}-u_{2}\right\|_{2 p} \mathrm{~d} s \\
\leq & t^{\alpha}(4 \pi)^{-\frac{3(p-1)}{4 p}} C_{f} \max \left\{2^{p-2}, 1\right\}\left(2 R_{2}+2 T^{(p-1) \alpha}\|v\|_{\infty, 2 p}^{p-1}\right) \\
& \times \int_{0}^{t}(t-s)^{-\frac{3(p-1)}{4 p}} s^{-(p-1) \alpha}\left\|u_{1}-u_{2}\right\|_{2 p} \mathrm{~d} s \\
\leq & t^{\alpha}(4 \pi)^{-\frac{3(p-1)}{4 p}} C_{f} \max \left\{2^{p-2}, 1\right\}\left(2 R_{2}+2 T^{(p-1) \alpha}\|v\|_{\infty, 2 p}^{p-1}\right) \\
& \times \int_{0}^{t}(t-s)^{-\frac{3(p-1)}{4 p}} s^{\alpha}\left\|u_{1}-u_{2}\right\|_{2 p} s^{-p \alpha} \mathrm{d} s \\
\leq & (4 \pi)^{-\frac{3(p-1)}{4 p}} C_{f} \max \left\{2^{p-2}, 1\right\}\left(2 R_{2}+2 T^{(p-1) \alpha}\|v\|_{\infty, 2 p}^{p-1}\right)\left\|u_{1}-u_{2}\right\|_{Y_{T}} \\
& \times t^{\alpha} \int_{0}^{t}(t-s)^{-\frac{3(p-1)}{4 p}} s^{-p \alpha} \mathrm{d} s,
\end{aligned}
$$

noticing that here we use the fact: $u_{1}, u_{2} \in B$, i.e. $s^{(p-1) \alpha}\left\|u_{1}\right\|_{2 p}^{p-1}, s^{(p-1) \alpha}\left\|u_{2}\right\|_{2 p}^{p-1} \leq R_{2}$. We consider the last term on the RHS of the previous inequality

$$
\begin{aligned}
& t^{\alpha} \int_{0}^{t}(t-s)^{-\frac{3(p-1)}{4 p}} s^{-p \alpha} \mathrm{d} s \\
= & \int_{0}^{t} t^{1+\alpha-p \alpha-\frac{3(p-1)}{4 p}}\left(1-\frac{s}{t}\right)^{-\frac{3(p-1)}{4 p}}\left(\frac{s}{t}\right)^{-p \alpha} d\left(\frac{s}{t}\right) \\
= & t^{1+\alpha-p \alpha-\frac{3(p-1)}{4 p}} \int_{0}^{1}(1-\nu)^{-\frac{3(p-1)}{4 p}} \nu^{-p \alpha} \mathrm{d} \nu \\
= & t^{1+\alpha-p \alpha-\frac{3(p-1)}{4 p}}\left[\int_{0}^{\frac{1}{2}}(1-\nu)^{-\frac{3(p-1)}{4 p}} \nu^{-p \alpha} \mathrm{d} \nu+\int_{\frac{1}{2}}^{1}(1-\nu)^{-\frac{3(p-1)}{4 p}} \nu^{-p \alpha} \mathrm{d} \nu\right] .
\end{aligned}
$$

A simple computation gives

$$
\int_{0}^{\frac{1}{2}}(1-\nu)^{-\frac{3(p-1)}{4 p}} \nu^{-p \alpha} \mathrm{d} \nu \leq \int_{0}^{\frac{1}{2}} \nu^{-\frac{3(p-1)}{4 p}-p \alpha} \mathrm{d} \nu=\frac{2^{\frac{3(p-1)}{4 p}+p \alpha-1}}{1-\frac{3(p-1)}{4 p}-p \alpha},
$$

and

$$
\int_{\frac{1}{2}}^{1}(1-\nu)^{-\frac{3(p-1)}{4 p}} \nu^{-p \alpha} \mathrm{d} \nu \leq \int_{\frac{1}{2}}^{1}(1-\nu)^{-\frac{3(p-1)}{4 p}-p \alpha} \mathrm{d} \nu=\frac{2^{\frac{3(p-1)}{4 p}+p \alpha-1}}{1-\frac{3(p-1)}{4 p}-p \alpha}
$$


As a consequence to these estimates

$$
\begin{aligned}
& t^{\alpha} \int_{0}^{t}\left\|S(t-s)\left(f\left(u_{1}+v\right)-f\left(u_{2}+v\right)\right)\right\|_{2 p} \mathrm{~d} s \\
\leq & (4 \pi)^{-\frac{3(p-1)}{4 p}} C_{f} \max \left\{2^{p-2}, 1\right\}\left(2 R_{2}+2 T^{(p-1) \alpha}\|v\|_{\infty, 2 p}^{p-1}\right) \\
& \times \frac{2^{\frac{3(p-1)}{4 p}+p \alpha}}{1-\frac{3(p-1)}{4 p}-p \alpha} T^{1+\alpha-p \alpha-\frac{3(p-1)}{4 p}}\left\|u_{1}-u_{2}\right\|_{Y_{T}} \\
= & (4 \pi)^{-\frac{3(p-1)}{4 p}} C_{f} \max \left\{2^{p-2}, 1\right\}\left(2 R_{2}+2 T^{(p-1) \alpha}\|v\|_{\infty, 2 p}^{p-1}\right) \\
& \times \frac{2^{\frac{3(p-1)}{4 p}+p \alpha}}{1-\frac{3(p-1)}{4 p}-p \alpha} T^{\frac{p+3}{8 p}}\left\|u_{1}-u_{2}\right\|_{Y_{T} .}
\end{aligned}
$$

We put $\quad C_{1}=(4 \pi)^{-\frac{3(p-1)}{4 p}} C_{f} \max \left\{2^{p-2}, 1\right\}\left(2 R_{2}+2 T^{(p-1) \alpha}\|v\|_{\infty, 2 p}^{p-1}\right) \frac{2^{\frac{3(p-1)}{4 p}+p \alpha}}{1-\frac{3(p-1)}{4 p}-p \alpha} T^{\frac{p+3}{8 p}}, \quad$ then $\quad C_{1} \leq$ $(4 \pi)^{-\frac{3(p-1)}{4 p}} C_{f} \max \left\{2^{p-2}, 1\right\}\left(2 R_{2}+2 T_{*}^{(p-1) \alpha}\|v\|_{\infty, 2 p}^{p-1}\right) \frac{2^{\frac{3(p-1)}{4 p}+p \alpha}}{1-\frac{3(p-1)}{4 p}-p \alpha} T_{*}^{\frac{p+3}{8 p}} \leq(4 \pi)^{-\frac{3(p-1)}{4 p}} C_{f} \max \left\{2^{p-2}, 1\right\}\left(2 R_{2}+\right.$ $\left.2\|v\|_{\infty, 2 p}^{p-1}\right) \frac{2^{\frac{3(p-1)}{4 p}+p \alpha}}{1-\frac{3(p-1)}{4 p}-p \alpha} T_{*}^{\frac{p+3}{8 p}} \leq \frac{1}{2}$. This implies that $\Phi$ is a contraction in the Banach space $Y_{T}$.

Choosing $u_{2}$ to be 0 in this estimate, we obtain also that $\left\|\Phi\left(u_{1}\right)-\Phi(0)\right\|_{Y_{T}} \leq C_{1}\left\|u_{1}\right\|_{Y_{T}} \leq \frac{1}{2} R_{2}$. Moreover, the estimate

$$
\begin{aligned}
t^{\alpha} \int_{0}^{t}\|S(t-s)(f(v))\|_{2 p} \mathrm{~d} s & \leq \int_{0}^{t}(4 \pi(t-s))^{-\frac{3(p-1)}{4 p}}\|f(v)\|_{2} \mathrm{~d} s \\
& \leq \int_{0}^{t}(t-s)^{-\frac{3(p-1)}{4 p}}\|f(v)\|_{2} \mathrm{~d} s \leq \frac{1}{2} R_{2}
\end{aligned}
$$

implies that $\left\|\Phi\left(u_{1}\right)\right\|_{Y_{T}} \leq R_{2}$. Which means that $\Phi$ is a contraction from a complete metric space to itself and it admits a unique fixed-point $u$ in this set according to the Banach Theorem.

Step 2. We prove that $u \in L^{\infty}(\mathcal{O} \times(0, T))$.

Step 2.1. We consider the case $v \in L^{\infty}\left(0, T, L^{2 p}(\mathcal{O})\right)$.

Firstly, we prove that $u \in L^{\infty}\left(0, T, L^{2 p}(\mathcal{O})\right)$. From $u(x, t)=\int_{0}^{t} S(t-s) f(u+v)(x, s) \mathrm{d} s$, we have the following inequality

$$
\begin{aligned}
\|u(., t)\|_{2 p} & \leq \int_{0}^{t}\|S(t-s) f(u+v)\|_{2 p} \mathrm{~d} s \leq \int_{0}^{t}(4 \pi(t-s))^{-\frac{3(p-1)}{4 p}}\|f(u+v)\|_{2} \mathrm{~d} s \\
& \leq \int_{0}^{t}(4 \pi(t-s))^{-\frac{3(p-1)}{4 p}}\left(\|f(u+v)-f(v)\|_{2}+\|f(v)\|_{2}\right) \mathrm{d} s .
\end{aligned}
$$

The computations

$$
\begin{aligned}
\|f(v)\|_{2} & \leq\|f(0)\|_{2}+\left\|f^{\prime}(\mu) v\right\|_{2} \leq\|f(0)\|_{2}+\left\|f^{\prime}(\mu)\right\|_{\frac{2 p}{p-1}}\|v\|_{2 p} \\
& \leq\|f(0)\|_{2}+\left\|C_{f}|v|^{p-1}\right\|_{\frac{2 p}{p-1}}\|v\|_{2 p}=\|f(0)\|_{2}+C_{f}\|v\|_{2 p}^{p}
\end{aligned}
$$


implies that $f(v)$ belongs to $L^{\infty}\left(0, T, L^{2}(\mathcal{O})\right)$. Combining this with the estimate of $\|u(., t)\|_{2 p}$, we obtain

$$
\begin{aligned}
\|u(x, t)\|_{2 p} \leq & \int_{0}^{t}(4 \pi(t-s))^{-\frac{3(p-1)}{4 p}}\left(\left\|f^{\prime}(v+\zeta)\right\|_{\frac{2 p}{p-1}}\|u\|_{2 p}+\|f(v)\|_{\infty, 2}\right) \mathrm{d} s \\
\leq & \int_{0}^{t}(4 \pi(t-s))^{-\frac{3(p-1)}{4 p}}\left(\left\|C_{f}|v+\zeta|^{p-1}\right\|_{\frac{2 p}{p-1}}\|u\|_{2 p}+\|f(v)\|_{\infty, 2}\right) \mathrm{d} s \\
\leq & \int_{0}^{t}(4 \pi(t-s))^{-\frac{3(p-1)}{4 p}}\left(\left\|C_{f} \max \left\{1,2^{p-2}\right\}\left(|v|^{p-1}+|u|^{p-1}\right)\right\|_{\frac{2 p}{p-1}}\|u\|_{2 p}\right. \\
& \left.+\|f(v)\| \|_{\infty, 2}\right) \mathrm{d} s \\
\leq & \int_{0}^{t}(4 \pi(t-s))^{-\frac{3(p-1)}{4 p}}\left[C_{f} \max \left\{1,2^{p-2}\right\}\left(\|v\|_{2 p}^{p-1}+\|u\|_{2 p}^{p-1}\right)\|u\|_{2 p}\right. \\
& \left.+\left.\|f(v)\|\right|_{\infty, 2}\right] \mathrm{d} s \\
\leq & {\left[\int_{0}^{t}(4 \pi(t-s))^{-\frac{3(p-1) l_{1}}{4 p}} \mathrm{~d} s\right]^{\frac{1}{l_{1}}} } \\
& \times\left[\int_{0}^{t}\left[C_{f} \max \left\{1,2^{p-2}\right\}\left(\|v\|_{\infty, 2 p}^{p-1}+\|u\|_{2 p}^{p-1}\right)\|u\|_{2 p}+\|f(v)\|_{\infty, 2}\right]^{l_{2}} \mathrm{~d} s\right]^{\frac{1}{l_{2}}}
\end{aligned}
$$

notice that $l_{1}, l_{2}>0, \frac{1}{l_{1}}+\frac{1}{l_{2}}=1$ and $l_{1}<\frac{4 p}{3(p-1)}$.

The inequality

$$
\int_{0}^{t}(t-s)^{-\frac{3(p-1) l_{1}}{4 p}} \mathrm{~d} s=-\left.\frac{(t-s)^{1-\frac{3(p-1) l_{1}}{4 p}}}{1-\frac{3(p-1) l_{1}}{4 p}}\right|_{0} ^{t}=\frac{t^{1-\frac{3(p-1) l_{1}}{4 p}}}{1-\frac{3(p-1) l_{1}}{4 p}} \leq \frac{T^{1-\frac{3(p-1) l_{1}}{4 p}}}{1-\frac{3(p-1) l_{1}}{4 p}}
$$

leads to

$$
\begin{aligned}
& \|u\|_{2 p} \leq\left(\frac{T^{1-\frac{3(p-1) l_{1}}{4 p}}}{1-\frac{3(p-1) l_{1}}{4 p}}\right)^{\frac{1}{l_{1}}}(4 \pi)^{-\frac{3(p-1)}{4 p}} \\
& \times\left[\int_{0}^{t}\left[C_{f} \max \left\{1,2^{p-2}\right\}\left(\|v\|_{\infty, 2 p}^{p-1}+\|u\|_{2 p}^{p-1}\right)\|u\|_{2 p}+\|f(v)\|_{\infty, 2}\right]^{l_{2}} \mathrm{~d} s\right]^{\frac{1}{l_{2}}} .
\end{aligned}
$$

Put $U(t)=\|u(., t)\|_{2 p}^{l_{2}}$, the inequality (3.8) becomes

$$
\begin{aligned}
U(t) \leq & \left(\frac{T^{1-\frac{3(p-1) l_{1}}{4 p}}}{1-\frac{3(p-1) l_{1}}{4 p}}\right)^{\frac{l_{2}}{l_{1}}}(4 \pi)^{-\frac{3(p-1) l_{2}}{4 p}} \\
& \times \int_{0}^{t}\left[C_{f} \max \left\{1,2^{p-2}\right\}\left(\|v\|_{\infty, 2 p}^{p-1}+U(s)^{\frac{p-1}{l_{2}}}\right) U(s)^{\frac{1}{l_{2}}}+\|f(v)\|_{\infty, 2}\right]^{l_{2}} \mathrm{~d} s .
\end{aligned}
$$

We denote $V(t)=\left(\frac{T^{1-\frac{3(p-1) l_{1}}{4 p}}}{1-\frac{3(p-1) l_{1}}{4 p}}\right)^{\frac{l_{2}}{l_{1}}}(4 \pi)^{-\frac{3(p-1) l_{2}}{4 p}} \int_{0}^{t}\left[C_{f} \max \left\{1,2^{p-2}\right\} \quad\left(\|v\|_{\infty, 2 p}^{p-1}+U(s)^{\frac{p-1}{l_{2}}}\right) \quad U(s)^{\frac{1}{l_{2}}}\right.$ $\left.+\|f(v)\|_{\infty, 2}\right]^{l_{2}} \mathrm{~d} s$, then $V^{\prime}(t)=\left(\frac{T^{1-\frac{3(p-1) l_{1}}{4 p}}}{1-\frac{3(p-1) l_{1}}{4 p}}\right)^{\frac{l_{2}}{l_{1}}}(4 \pi)^{-\frac{3(p-1) l_{2}}{4 p}}\left[C_{f} \max \left\{1,2^{p-2}\right\}\left(\|v\|_{\infty, 2 p}^{p-1}+U(t)^{\frac{p-1}{l_{2}}}\right) U(t)^{\frac{1}{l_{2}}}+\right.$ $\left.\|f(v)\|_{\infty, 2}\right]^{l_{2}}$. Since $U(t) \leq V(t)$, then $V^{\prime}(t) \leq\left(\frac{T^{1-\frac{3(p-1) l_{1}}{4 p}}}{1-\frac{3(p-1) l_{1}}{4 p}}\right)^{\frac{l_{2}}{l_{1}}}(4 \pi)^{-\frac{3(p-1) l_{2}}{4 p}}\left[C_{f} \max \left\{1,2^{p-2}\right\}\left(\|v\|_{\infty, 2 p}^{p-1}\right.\right.$ $\left.\left.+V(s)^{\frac{p-1}{l_{2}}}\right) V(s)^{\frac{1}{l_{2}}}+\|f(v)\|_{\infty, 2}\right]^{l_{2}}$. 
Basing on the notations in (2.5), we put

$$
G(r)=\int_{0}^{r} \frac{(4 \pi)^{\frac{3(p-1) l_{2}}{4 p}} \mathrm{~d} \zeta}{\left(\frac{T^{1-\frac{3(p-1) l_{1}}{4 p}}}{1-\frac{3(p-1) l_{1}}{4 p}}\right)^{\frac{l_{2}}{l_{1}}}\left[C_{f} \max \left\{1,2^{p-2}\right\}\left(\|v\|_{\infty, 2 p}^{p-1}+\zeta^{\frac{p-1}{l_{2}}}\right) \zeta^{\frac{1}{l_{2}}}+\|f(v)\|_{\infty, 2}\right]^{l_{2}}},
$$

then $G$ is an increasing function on $[0,+\infty)$ and $G^{\prime}(V(t)) \leq 1$. Hence $G(V(t)) \leq t+G(V(0))=t+G(0)=t \leq T_{*}$ and $U(t) \leq V(t) \leq G^{-1}\left(T_{*}\right)$. Finally, $\|u(., t)\|_{2 p} \leq G^{-1}\left(T_{*}\right)^{\frac{1}{l_{2}}}$. We have proved that $u \in L^{\infty}\left(0, T, L^{2 p}(\mathcal{O})\right)$ by proving a New Gronwall Inequality on $U(t)$.

Secondly, we prove that $u \in L^{\infty}\left(0, T, L^{\infty}(\mathcal{O})\right)$. Using Proposition 48.4, page 441, [21], we get the following estimate

$$
\begin{aligned}
\|u(., t)\|_{\infty} \leq & \int_{0}^{t}\|S(t-s) f(u+v)(s)\|_{\infty} \mathrm{d} s \leq \int_{0}^{t}(4 \pi(t-s))^{-\frac{3}{4}}\|f(u+v)(s)\|_{2} \mathrm{~d} s \\
\leq & \int_{0}^{t}(4 \pi(t-s))^{-\frac{3}{4}}\left(\|f(u+v)(s)-f(v)\|_{2}+\|f(v)\|_{2}\right) \mathrm{d} s \\
\leq & \int_{0}^{t}(4 \pi(t-s))^{-\frac{3}{4}}\left[C_{f} \max \left\{1,2^{p-2}\right\}\left(\|u\|_{2 p}^{p-1}+\|v\|_{\infty, 2 p}^{p-1}\right)\|u\|_{2 p}+\|f(v)\|_{\infty, 2}\right] \mathrm{d} s \\
\leq & \int_{0}^{t}(4 \pi(t-s))^{-\frac{3}{4}}\left[C_{f} \max \left\{1,2^{p-2}\right\}\left(G^{-1}\left(T_{*}\right)^{\frac{p-1}{l_{2}}}+\|v\|_{\infty, 2 p}^{p-1}\right) G^{-1}\left(T_{*}\right)^{\frac{1}{l_{2}}}\right. \\
& \left.+\|f(v)\|_{\infty, 2}\right] \mathrm{d} s \\
\leq & \pi^{-\frac{3}{4}}\left(4 T_{*}\right)^{\frac{1}{4}}\left[C_{f} \max \left\{1,2^{p-2}\right\}\left(G^{-1}\left(T_{*}\right)^{\frac{p-1}{l_{2}}}+\|v\|_{\infty, 2 p}^{p-1}\right) G^{-1}\left(T_{*}\right)^{\frac{1}{l_{2}}}\right. \\
& \left.+\|f(v)\|_{\infty, 2}\right],
\end{aligned}
$$

which deduces $u \in L^{\infty}(\mathcal{O} \times(0, T))$.

Step 2.2. For the case $v \in L^{\infty}\left(0, T, L^{\infty}(\mathcal{O})\right)$, we use exactly the same argument, but with the function $G(r)=$

$$
\int_{0}^{r} \frac{(4 \pi)^{\frac{3(p-1) l_{2}}{4 p}} \mathrm{~d} \zeta}{\left.\left(\frac{T^{1-\frac{3(p-1) l_{1}}{4 p}}}{1-\frac{3(p-1) l_{1}}{4 p}}\right)^{\frac{l_{2}}{l_{1}}}\left[C_{f} \max \left\{1,2^{p-2}\right\}\left(\left(M m(\mathcal{O})^{\frac{1}{2 p}}\right)^{p-1}\right)+\zeta^{\frac{p-1}{l_{2}}}\right) \zeta^{\frac{1}{l_{2}}}+\max _{|\zeta| \leq M}|f(\zeta)| m(\mathcal{O})^{\frac{1}{2}}\right]^{l_{2}}} .
$$

Then we have $\|u(., t)\|_{\infty} \leq \pi^{-\frac{3}{4}}\left(4 T_{*}\right)^{\frac{1}{4}}\left[C_{f} \max \left\{1,2^{p-2}\right\}\left(G^{-1}\left(T_{*}\right)^{\frac{p-1}{l_{2}}}+\left(M m(\mathcal{O})^{\frac{1}{2 p}}\right)^{p-1}\right) G^{-1}\left(T_{*}\right)^{\frac{p-1}{l_{2}}}\right.$ $\left.+\max _{|\zeta| \leq M}|f(\zeta)| m(\mathcal{O})^{\frac{1}{2}}\right]$ and $u \in L^{\infty}(\mathcal{O} \times(0, T))$.

Step 3. We prove that $u \in C\left([0, T], L^{2}(\mathcal{O})\right)$ and $u$ is also a solution of $(2.4)$. The proof in this step works well for both cases $v \in L^{\infty}\left(0, T, L^{2 p}(\mathcal{O})\right)$ and $v \in L^{\infty}\left(0, T, L^{\infty}(\mathcal{O})\right)$.

For $\epsilon$ positive,

$$
\begin{aligned}
u(., t+\epsilon)-u(., t) & =\int_{0}^{t+\epsilon} S(t+\epsilon-s) f(u+v)(., s) \mathrm{d} s-\int_{0}^{t} S(t-s) f(u+v)(., s) \mathrm{d} s \\
& =\int_{0}^{t}[S(t+\epsilon-s)-S(t-s)] f(u+v)(., s) \mathrm{d} s+\int_{t}^{t+\epsilon} S(t+\epsilon-s) f(u+v)(., s) \mathrm{d} s,
\end{aligned}
$$

which implies the following inequality

$$
\|u(., t+\epsilon)-u(., t)\|_{2} \leq \int_{0}^{t}\|[S(t+\epsilon-s)-S(t-s)] f(u+v)(., s)\|_{2} \mathrm{~d} s+\int_{t}^{t+\epsilon}\|S(t+\epsilon-s) f(u+v)(., s)\|_{2} \mathrm{~d} s .
$$


We firstly estimate the second term on the RHS of the previous inequality. Due to Proposition 48.4 ([21], p. 441),

$$
\int_{t}^{t+\epsilon}\|S(t+\epsilon-s) f(u+v)(., s)\|_{2} \mathrm{~d} s \leq \int_{t}^{t+\epsilon}\|f(u+v)(., s)\|_{2} \mathrm{~d} s .
$$

Since $\int_{t}^{t+\epsilon}\|f(u+v)(., s)\|_{2} \mathrm{~d} s$ tends to 0 as $\epsilon$ tends to $0, \int_{t}^{t+\epsilon}\|S(t+\epsilon-s) f(u+v)(., s)\|_{2} \mathrm{~d} s$ tends to 0 as $\epsilon$ tends to 0.

Now, we estimate the first term. Noticing that $\|[S(t+\epsilon-s)-S(t-s)] f(u+v)(., s)\|_{2}$ tends to 0 as $\epsilon$ tends to 0 for all $s$ in $[0, t]$, and

$$
\begin{aligned}
& \|[S(t+\epsilon-s)-S(t-s)] f(u+v)(., s)\|_{2} \\
\leq & \|S(t+\epsilon-s) f(u+v)(., s)\|_{2}+\|S(t-s) f(u+v)(., s)\|_{2} \leq 2\|f(u+v)(., s)\|_{2},
\end{aligned}
$$

where $f(u+v)$ can be proved to belong to $L^{\infty}\left(0, T, L^{2}(\mathcal{O})\right)$ by using the same argument as before; due to the Lebesgue Dominated Convergence Theorem, we deduce that

$$
\lim _{\epsilon \rightarrow 0} \int_{0}^{t}\|[S(t+\epsilon-s)-S(t-s)] f(u+v)(., s)\|_{2} \mathrm{~d} s=0 .
$$

Therefore $\lim _{\epsilon \rightarrow 0}\|u(., t+\epsilon)-u(., t)\|_{2}=0$, or $u \in C\left([0, T], L^{2}(\mathcal{O})\right)$.

Since $v \in C\left([0, T], L^{2}(\mathcal{O})\right), u+v \in C\left([0, T], L^{2}(\mathcal{O})\right)$. Which implies $f(u+v) \in C\left([0, T], L^{2}(\mathcal{O})\right)$; and by Lemma $3.2, u$ is also a solution of $(2.4)$.

\section{Proof of the Existence RESUlts For SEMilinear heAt EQUATion IN A CYLINDRICAL BOUNDED DOMAIN - THEOREM 2.3}

First, we consider the following equation

$$
\begin{cases}\partial_{t} v-\Delta v=0 & \text { in } \Omega \times\left(0, T_{*}\right), \\ v=g & \text { on } \partial \Omega \\ v(., 0)=u_{0}(.) & \text { on } \bar{\Omega}\end{cases}
$$

We recall the following definition:

Definition 4.1 ([7], Def. c p. 69). Let $\omega$ be a domain in $\mathbb{R}^{N}$, we say that $\partial \omega \times(0, T]$ has the outside strong sphere property if for every $Q=\left(x_{0}, t_{0}\right)$ in $\partial \omega \times(0, T]$, there exists a ball $K$ with center $(\bar{x}, \bar{t})$ such that $K \cap(\bar{\omega} \times[0, T])=\{Q\}$ and $|\bar{x}-x| \geq \mu(Q)>0$, for every $(x, t)$ in $\bar{\omega} \times[0, T],\left|t-t_{0}\right|<\epsilon$, where $\epsilon$ is a constant independent of $Q$.

We will prove that, in fact $\partial \Omega \times(0, T]$ has the outside strong sphere property.

Let $Q=\left(x_{0}, t_{0}\right)$ be on $\partial \Omega \times(0, T]$. Since $D$ is smooth, there exists $\bar{x}$ and a constant $r>0$ such that $K=\bar{B}_{\mathbb{R}^{N}}(\bar{x}, r) \cap \bar{\Omega}=\left\{x_{0}\right\}$ and $\left\|\bar{x}-x_{0}\right\|_{\mathbb{R}^{N}}=r$.

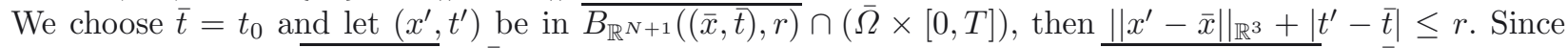
$\left\|x^{\prime}-\bar{x}\right\|_{\mathbb{R}^{N}} \leq r ; x^{\prime} \in \overline{B_{\mathbb{R}^{N}}(\bar{x}, r)} \cap \bar{\Omega}$ and $x^{\prime}=x_{0}$. We infer that $t^{\prime}=t_{0}$ and $\overline{B_{\mathbb{R}^{N+1}}((\bar{x}, \bar{t}), r)} \cap(\bar{\Omega} \times[0, T])=$ $\left\{\left(x_{0}, t_{0}\right)\right\}$. For $(x, t) \in \bar{\Omega} \times[0, T],\|x-\bar{x}\|_{\mathbb{R}^{N}} \geq r>0$. Consequently, $\partial \Omega \times(0, T]$ has the outside strong sphere property.

By Theorem 8 ([7], p. 69), we can conclude that $\partial \Omega \times(0, T]$ has local barriers. Due to Theorem 5 page 123, [7], there exists a unique solution $v$ to the problem (4.1), and $v \in C^{1,2}((0, T] \times \Omega) \cap C(\bar{\Omega} \times[0, T])$. According to Theorem 10 page $72,[7], v \in C^{\infty}(\Omega \times(0, T))$. 
Due to the Weak Maximum Principle ([6], p. 368): $\max _{\bar{\Omega} \times[0, T]} v=\max _{\partial(\Omega \times(0, T))} v$, and $\min _{\bar{\Omega} \times[0, T]} v=$ $\min _{\partial(\Omega \times(0, T))} v$. Which gives $v<M$ and $v>-M$ on $\bar{\Omega} \times[0, T]$ since $M>\max \left\{\left\|u_{0}\right\|_{\infty},\|g\|_{\infty}\right\}$. Then $\|v\|_{L^{\infty}(\Omega \times(0, T))}<M$.

Subtracting (4.1) and (2.7), and put $w=u-v$, we have the following equations

$$
\begin{cases}\partial_{t} w-\Delta w=f(w+v) & \text { in } \Omega \times(0, T), \\ w(., .)=0 & \text { on } \partial \Omega, \\ w(., 0)=0 & \text { on } \bar{\Omega} .\end{cases}
$$

According to Theorem 2.2, (4.2) has a solution $w$ satisfying $w \in L^{\infty}(\Omega \times(0, T)) \cap C\left([0, T], L^{2}(\Omega)\right) \cap$ $L^{2}\left(0, T, H_{0}^{1}(\Omega)\right)$ and $\partial_{t} w \in L^{2}\left(0, T, L^{2}(\Omega)\right)$. Moreover, $\|w\|_{\infty, \infty} \leq \pi^{-\frac{3}{4}}\left(4 T_{*}\right)^{\frac{1}{4}}\left[C_{f} \max \left\{1,2^{p-2}\right\}\left(G^{-1}\left(T_{*}\right)^{\frac{p-1}{l_{2}}}\right.\right.$ $\left.+\left(\left(M m(\Omega)^{\frac{1}{2 p}}\right)^{p-1}\right) G^{-1}\left(T_{*}\right)^{\frac{p-1}{l_{2}}}+\max _{|\zeta| \leq M}|f(\zeta)| m(\Omega)^{\frac{1}{2}}\right]$, where

$$
G(r)=\int_{0}^{r} \frac{(4 \pi)^{\frac{3(p-1) l_{2}}{4 p}} \mathrm{~d} \zeta}{\left(\frac{T^{1-\frac{3(p-1) l_{1}}{4 p}}}{1-\frac{3(p-1) l_{1}}{4 p}}\right)^{\frac{l_{2}}{l_{1}}}\left[C_{f} \max \left\{1,2^{p-2}\right\}\left(\left(M m(\Omega)^{\frac{1}{2 p}}\right)^{p-1}+\zeta^{\frac{p-1}{l_{2}}}\right) \zeta^{\frac{1}{l_{2}}}+\max _{|\zeta| \leq M}|f(\zeta)| m(\Omega)^{\frac{1}{2}}\right]^{l_{2}}} .
$$

Hence (2.7) has a solution $u$ satisfying $u \in L^{\infty}(\Omega \times(0, T)) \cap C\left([0, T], L^{2}(\Omega)\right) \cap L^{2}\left(0, T, H_{0}^{1}(\Omega)\right)$ and $\partial_{t} u \in L^{2}\left(0, T, L^{2}(\Omega)\right)$. Moreover, $\|u\|_{\infty, \infty} \leq M_{*}:=M+\pi^{-\frac{3}{4}}\left(4 T_{*}\right)^{\frac{1}{4}}\left[C_{f} \max \left\{1,2^{p-2}\right\}\left(G^{-1}\left(T_{*}\right)^{\frac{p-1}{l_{2}}}\right.\right.$ $\left.+\left(\left(M m(\Omega)^{\frac{1}{2 p}}\right)^{p-1}\right) G^{-1}\left(T_{*}\right)^{\frac{p-1}{l_{2}}}+\max _{|\zeta| \leq M}|f(\zeta)| m(\Omega)^{\frac{1}{2}}\right]$.

Using the results in Section VI.8 of [15] for the equation $\partial_{t} u-\Delta u=g$ in $\Omega \times(0, T)$, where $g=f(u+v)$, $g \in L^{\infty}(\Omega \times(0, T))$, we can conclude that $u$ is continuous on $\overline{\Omega \times(0, T)}$.

\section{Proof of the Well-Posedness And Convergence Properties OF THE ALGORITHMS - THEOREMS 2.5 AND 2.6}

\subsection{The well-posedness of the algorithm - Proof of Theorem 2.5}

We consider equation $(2.9)$

$$
\begin{cases}\partial_{t} \phi_{M}-\Delta \phi_{M}=f\left(\phi_{M}\right) & \text { in } \Omega \times\left(0, T_{0}\right), \\ \phi_{M}(., .)=M & \text { on } \partial \Omega \times\left[0, T_{0}\right], \\ \phi_{M}(., 0)=M & \text { on } \bar{\Omega} .\end{cases}
$$

Since $f(x)$ is positive for all $x$ in $\mathbb{R}$, then $\partial_{t} \phi_{M}-\Delta \phi_{M} \geq 0$ and $\partial_{t}\left(\phi_{M}-M\right)-\Delta\left(\phi_{M}-M\right) \geq 0$. Since $\phi_{M}=M$ on $\left(\partial \Omega \times\left[0, T_{0}\right]\right) \cup(\bar{\Omega} \cap\{0\})$, then $\phi_{M} \geq M$ on $\overline{\Omega \times\left[0, T_{0}\right]}$ according to the Maximum Principle.

We will prove that the algorithm is well posed for $T<T^{*}$ by recursion.

For $k=1$, we consider the problem on the $j$ th domain

$$
\begin{cases}\partial_{t} u_{j}^{1}-\Delta u_{j}^{1}=f\left(u_{j}^{1}\right) & \text { in } \Omega_{j} \times(0, T), \\ u_{j}^{1}\left(\cdot, a_{j}, \cdot\right)=u_{j-1}^{0}\left(\cdot, a_{j}, \cdot\right) & \text { in } D \times(0, T), \\ u_{j}^{1}\left(\cdot, b_{j}, \cdot\right)=u_{j+1}^{0}\left(\cdot, b_{j}, \cdot\right) & \text { in } D \times(0, T), \\ u_{j}^{1}(., .)=g(., .) & \text { on } \partial D \times\left[a_{j}, b_{j}\right] \times[0, T], \\ u_{j}^{1}(., 0)=u_{0}(.) & \text { on } \overline{\Omega_{j}} .\end{cases}
$$


According to Theorem 2.3, the equation (5.2) has a solution $u_{j}^{1}$ satisfying $u_{j}^{1} \in L^{\infty}\left(\Omega_{j} \times(0, T)\right) \cap$ $C\left([0, T], L^{2}\left(\Omega_{j}\right)\right) \cap L^{2}\left(0, T, H_{0}^{1}\left(\Omega_{j}\right)\right), \partial_{t} u_{j}^{1} \in L^{2}\left(0, T, L^{2}\left(\Omega_{j}\right)\right)$ and $u_{j}^{1}$ is continuous on $\overline{\Omega_{j} \times(0, T)}$. The fact that $\left.u_{j}^{1} \in C^{1,2}((0, T) \times \Omega)\right)$ then follows.

Since $\partial_{t}\left(\phi_{M}-u_{j}^{1}\right)-\Delta\left(\phi_{M}-u_{j}^{1}\right)=f\left(\phi_{M}\right)-f\left(u_{j}^{1}\right) ; \partial_{t}\left(\phi_{M}-u_{j}^{1}\right)-\Delta\left(\phi_{M}-u_{j}^{1}\right)-\left(\phi_{M}-u_{j}^{1}\right) c(x, t)=0$, where

$$
c(x, t)= \begin{cases}\frac{f\left(\phi_{M}\right)-f\left(u_{j}^{1}\right)}{\phi_{M}-u_{j}^{1}} & \text { if } \phi_{M} \neq u_{j}^{1}, \\ f^{\prime}\left(\phi_{M}\right) & \text { otherwise. }\end{cases}
$$

We notice that $c \in C\left(\overline{\Omega_{j} \times(0, T)}\right)$ and $u_{j}^{1} \leq M \leq \phi_{M}$ on $\left(\partial \Omega_{j} \times[0, T]\right) \cup\left(\bar{\Omega}_{j} \times\{0\}\right)$. Then it follows by the Maximum Principle that $\phi_{M} \geq u_{j}^{1}$ on $\overline{\Omega_{j} \times(0, T)}$.

Since $\partial_{t}\left(u_{j}^{1}+M\right)-\Delta\left(u_{j}^{1}+M\right)-\left(f\left(u_{j}^{1}\right)-f(-M)\right)=f(-M) \geq 0$ and $u_{j}^{1} \geq-M$ on $\left(\partial \Omega_{j} \times[0, T]\right) \cup\left(\bar{\Omega}_{j} \times\{0\}\right)$; the Maximum Principle then implies that $u_{j}^{1} \geq-M$ on $\overline{\Omega_{j} \times(0, T)}$. Consequently, $\phi_{M} \geq u_{j}^{1} \geq-M$ on $\overline{\Omega_{j} \times(0, T)}$, $\forall j=\overline{1, I}$.

Suppose that the algorithm is well posed up to step $k=m$, and moreover $\phi_{M} \geq u_{j}^{i} \geq-M$ on $\overline{\Omega_{j} \times(0, T)}$ $\forall j=\overline{1, I}, i=\overline{1, m}$, using the same argument as above we can see that the algorithm is well posed for $k=m+1$ and $\phi_{M} \geq u_{j}^{m+1} \geq-M$ on $\overline{\Omega_{j} \times(0, T)}, \forall j=\overline{1, I}$.

Therefore, the algorithm is well posed for all $k \in \mathbb{N}$ and we have also $\phi_{M} \geq u_{j}^{k} \geq-M$ on $\overline{\Omega_{j} \times(0, T)}$, $\forall j=\overline{1, I}$.

\subsection{The convergence of the algorithm - Proof of Theorem 2.6}

We divide the proof into several steps.

Step 1. The Exponential Decay Error Estimates.

On the $j$ th domain, at the $k$ th iteration, we consider the equation

$$
\begin{cases}\partial_{t} e_{j}^{k}-\Delta e_{j}^{k}=f\left(u_{j}^{k}\right)-f(u) & \text { in } \Omega_{j} \times(0, T), \\ e_{j}^{k}\left(\cdot, a_{j}, \cdot\right)=e_{j-1}^{k-1}\left(\cdot, a_{j}, \cdot\right) & \text { in } D \times(0, T), \\ e_{j}^{k}\left(\cdot, b_{j}, \cdot\right)=e_{j+1}^{k-1}\left(\cdot, b_{j}, \cdot\right) & \text { in } D \times(0, T), \\ e_{j}^{k}(., .)=0 & \text { on } \partial D \times\left[a_{j}, b_{j}\right] \times[0, T], \\ e_{j}^{k}(., 0)=0 & \text { on } \bar{\Omega}_{j} .\end{cases}
$$

Since the algorithm is well posed, according to Definition 2.4, the set $\left\{u_{j}^{k} \mid j=\overline{1, I}, k \in \mathbb{N}\right\}$ is bounded by a constant $C_{1}$. This implies $\left\{e_{j}^{k} \mid j=\overline{1, I}, k \in \mathbb{N}\right\}$ is bounded by a constant $C_{2}$, where $C_{2}=C_{1}+\|u\|_{C(\overline{\Omega \times(0, T)})}$.

We consider the Controlling Function $\Phi(x, t)=P\left(e_{j}^{k}\right) \exp \left(\beta\left(z-a_{j}\right)-\gamma t\right)$, and $\mathcal{L}(\Phi)=\partial_{t} \Phi-\Delta \Phi+2 \beta \partial_{z} \Phi$, where $\beta$ and $\gamma$ are constants to be chosen later in the next steps and $z$ denotes the variable in the direction of $(a, b)$. We now prove that if we choose $(\beta, \gamma)$ such that $\gamma-\beta^{2}$ is large enough, $\mathcal{L}(\Phi)$ is negative.

A simple computation gives

$$
\begin{aligned}
\mathcal{L}(\Phi)= & \left(\partial_{t} e_{j}^{k}-\Delta e_{j}^{k}\right) P^{\prime}\left(e_{j}^{k}\right) \exp \left(\beta\left(z-a_{j}\right)-\gamma t\right) \\
& +\left(\beta^{2}-\gamma\right) P\left(e_{j}^{k}\right) \exp \left(\beta\left(z-a_{j}\right)-\gamma t\right)-\left(\nabla e_{j}^{k}\right)^{2} P^{\prime \prime}\left(e_{j}^{k}\right) \exp \left(\beta\left(z-a_{j}\right)-\gamma t\right) .
\end{aligned}
$$


By (5.3), the previous computation leads to

$$
\begin{aligned}
\mathcal{L}(\Phi)= & \left(f\left(u_{j}^{k}\right)-f(u)\right) P^{\prime}\left(e_{j}^{k}\right) \exp \left(\beta\left(z-a_{j}\right)-\gamma t\right) \\
& +\left(\beta^{2}-\gamma\right) P\left(e_{j}^{k}\right) \exp \left(\beta\left(z-a_{j}\right)-\gamma t\right)-\left(\nabla e_{j}^{k}\right)^{2} P^{\prime \prime}\left(e_{j}^{k}\right) \exp \left(\beta\left(z-a_{j}\right)-\gamma t\right),
\end{aligned}
$$

which implies

$$
\mathcal{L}(\Phi) \leq\left[\left(f\left(u_{j}^{k}\right)-f(u)\right) P^{\prime}\left(e_{j}^{k}\right)+\left(\beta^{2}-\gamma\right) P\left(e_{j}^{k}\right)\right] \exp \left(\beta\left(z-a_{j}\right)-\gamma t\right) .
$$

We consider the term $\left(f\left(u_{j}^{k}\right)-f(u)\right) P^{\prime}\left(e_{j}^{k}\right)+\left(\beta^{2}-\gamma\right) P\left(e_{j}^{k}\right)$. By the Mean Value Theorem, $f\left(u_{j}^{k}(x, t)\right)-f(u(x, t))=$ $e_{j}^{k}(x, t) f^{\prime}(\zeta(x, t))$, where $\zeta(x, t)$ is a number lying between $u_{j}^{k}(x, t)$ and $u(x, t)$. We observe that $|\zeta(x, t)| \leq$ $\left|u_{j}^{k}(x, t)\right|+|u(x, t)| \leq C_{1}+\|u\|_{C(\overline{\Omega \times(0, T)})}=C_{2}$, which implies $\left|f^{\prime}(\zeta(x, t))\right|$ is bounded on $\overline{\Omega \times(0, T)}$.

We fix a pair $(x, t)$ in $\Omega \times(0, T)$ :

- If $P\left(e_{j}^{k}(x, t)\right)=0$, then $e_{j}^{k}=0$ and $P^{\prime}\left(e_{j}^{k}\right)=0$. That means $\left(f\left(u_{j}^{k}\right)-f(u)\right) P^{\prime}\left(e_{j}^{k}\right)+\left(\beta^{2}-\gamma\right) P\left(e_{j}^{k}\right)=0$.

- If $P\left(e_{j}^{k}(x, t)\right) \neq 0$, then

$$
\frac{\left(f\left(u_{j}^{k}\right)-f(u)\right) P^{\prime}\left(e_{j}^{k}\right)+\left(\beta^{2}-\gamma\right) P\left(e_{j}^{k}\right)}{P\left(e_{j}^{k}\right)}=\frac{e_{j}^{k}(x, t) P^{\prime}\left(e_{j}^{k}\right)}{P\left(e_{j}^{k}\right)} f^{\prime}(\zeta(x, t))+\left(\beta^{2}-\gamma\right) .
$$

Since $e_{j}^{k}$ is bounded by $C_{2},\left|\frac{e_{j}^{k}(x, t) P^{\prime}\left(e_{j}^{k}\right)}{P\left(e_{j}^{k}\right)}\right|$ is bounded by $K\left(C_{2}\right)$; which means $\frac{e_{j}^{k}(x, t) P^{\prime}\left(e_{j}^{k}\right)}{P\left(e_{j}^{k}\right)} f^{\prime}(\zeta(x, t))$ is bounded on $\overline{\Omega \times(0, T)}$ as $\left|f^{\prime}(\zeta(x, t))\right|$ is bounded. Since $P\left(e_{j}^{k}\right)$ is positive, then if we choose $(\beta, \gamma)$ such that $-\beta^{2}+\gamma$ is large enough, we can have that $\left(f\left(u_{j}^{k}\right)-f(u)\right) P^{\prime}\left(e_{j}^{k}\right)+\left(\beta^{2}-\gamma\right) P\left(e_{j}^{k}\right)$ is negative.

Consequently, if $\gamma-\beta^{2}$ is large enough, $\left(f\left(u_{j}^{k}\right)-f(u)\right) P^{\prime}\left(e_{j}^{k}\right)+\left(\beta^{2}-\gamma\right) P\left(e_{j}^{k}\right)$ is negative or $\mathcal{L}(\Phi)$ is negative.

When $\mathcal{L}(\Phi) \leq 0$, according to the Maximum Principle, the function $\Phi(x, t)$ can only attain its maximum values on the boundary $\partial \Omega \times[0, T]$ or $\bar{\Omega} \times\{0\}$. We have that $\Phi \geq 0$, and moreover $\Phi=0$ on $\bar{\Omega}_{j} \times\{0\}$ and on $\partial D \times\left[a_{j}, b_{j}\right] \times[0, T]$. Thus:

- If the maximum value(s) of $\Phi$ can be achieved on $\bar{\Omega}_{j} \times\{0\}$ and on $\partial D \times\left[a_{j}, b_{j}\right] \times[0, T]$, then $\Phi=0$ on $\overline{\Omega \times(0, T)}$; which means that $e_{j}^{k}=0$ on $\overline{\Omega \times(0, T)}$.

- If $e_{j}^{k} \neq 0$, then the maximum value(s) of $\Phi$ can be achieved only on $\bar{D} \times\left\{a_{j}\right\} \times[0, T]$ or on $\bar{D} \times\left\{b_{j}\right\} \times[0, T]$.

For $x$ in $\mathbb{R}^{N}$, denote that $x=(X, z)$ where $X \in \mathbb{R}^{N-1}$ and $z \in \mathbb{R}$, we now have the following exponential decay estimates for the errors $e_{j}^{k}$.

Case 1. $j=1$.

We have that $e_{1}^{k}=0$ on $\bar{D} \times\left\{a_{1}\right\} \times[0, T]$. Since $e_{1}^{k} \neq 0$, the maximum value(s) of $\Phi$ can only be achieved on $\bar{D} \times\left\{b_{1}\right\} \times[0, T]$ and for $(X, z, t) \in \overline{\Omega_{1} \times(0, T)}$

$$
\begin{aligned}
& P\left(e_{1}^{k}\right)(X, z, t) \exp \left(\beta\left(z-a_{1}\right)-\gamma t\right) \\
\leq & \max _{\bar{D} \times[0, T]}\left\{P\left(e_{1}^{k}\right)\left(X, b_{1}, t\right) \exp \left(\beta\left(b_{1}-a_{1}\right)-\gamma t\right)\right\} \\
= & \max _{\bar{D} \times[0, T]}\left\{P\left(e_{2}^{k-1}\right)\left(X, b_{1}, t\right) \exp \left(\beta S_{1}-\gamma t\right)\right\} .
\end{aligned}
$$

Case 2. $j=I$.

We have that $e_{I}^{k}=0$ on $\bar{D} \times\left\{b_{I}\right\} \times[0, T]$. Since $e_{I}^{k} \neq 0$, the maximum value(s) of $\Phi$ can only be achieved on $\bar{D} \times\left\{a_{I}\right\} \times[0, T]$ and for $(X, z, t) \in \overline{\Omega_{I} \times(0, T)}$

$$
\begin{aligned}
P\left(e_{I}^{k}\right)(X, z, t) \exp \left(\beta\left(z-a_{I}\right)-\gamma t\right) & \leq \max _{\bar{D} \times[0, T]}\left\{P\left(e_{I}^{k}\right)\left(X, a_{I}, t\right) \exp (-\gamma t)\right\} \\
& =\max _{\bar{D} \times[0, T]}\left\{P\left(e_{I-1}^{k-1}\right)\left(X, a_{I}, t\right) \exp (-\gamma t)\right\} .
\end{aligned}
$$


Case 3. $1<j<I$.

The maximum value(s) of $\Phi$ can be achieved on both $\bar{D} \times\left\{a_{j}\right\} \times[0, T]$ and $\bar{D} \times\left\{b_{j}\right\} \times[0, T]$ and for $(X, z, t) \in \overline{\Omega_{j} \times(0, T)}$

$$
\begin{aligned}
P\left(e_{j}^{k}\right)(X, z, t) \exp \left(\beta\left(z-a_{j}\right)-\gamma t\right) \leq & \max \left\{\max _{\bar{D} \times[0, T]}\left\{P\left(e_{j}^{k}\right)\left(X, b_{j}, t\right) \exp \left(\beta\left(b_{j}-a_{j}\right)-\gamma t\right)\right\},\right. \\
& \left.\max _{\bar{D} \times[0, T]}\left\{P\left(e_{j}^{k}\right)\left(X, a_{j}, t\right) \exp (-\gamma t)\right\}\right\} \\
= & \left.\max _{\max _{\bar{D} \times[0, T]}\left\{P\left(e_{j+1}^{k-1}\right)\left(X, b_{j}, t\right) \exp \left(\beta\left(b_{j}-a_{j}\right)-\gamma t\right)\right\},} \max \left\{P\left(e_{j-1}^{k-1}\right)\left(X, a_{j}, t\right) \exp (-\gamma t)\right\}\right\} .
\end{aligned}
$$

Step 2. Proof of convergence.

Step 2.1. Estimate of the right boundaries of the sub-domains. Consider the $I$ th domain, at the $k$ th step, (5.5) infers

$$
P\left(e_{I}^{k}(X, z, t)\right) \exp \left(\beta\left(z-a_{I}\right)-\gamma t\right) \leq \max _{\bar{D} \times[0, T]}\left\{P\left(e_{I}^{k}\left(X, a_{I}, t\right)\right) \exp (-\gamma t)\right\}
$$

Replace $z$ by $b_{I-1}$, we get

$$
P\left(e_{I}^{k}\left(X, b_{I-1}, t\right)\right) \exp \left(\beta\left(b_{I-1}-a_{I}\right)-\gamma t\right) \leq \max _{\bar{D} \times[0, T]}\left\{P\left(e_{I}^{k}\left(X, a_{I}, t\right)\right) \exp (-\gamma t)\right\} .
$$

Since $e_{I}^{k}\left(X, b_{I-1}, t\right)=e_{I-1}^{k+1}\left(X, b_{I-1}, t\right)$,

$$
P\left(e_{I-1}^{k+1}\left(X, b_{I-1}, t\right)\right) \exp \left(\beta\left(b_{I-1}-a_{I}\right)-\gamma t\right) \leq \max _{\bar{D} \times[0, T]}\left\{P\left(e_{I}^{k}\left(X, a_{I}, t\right)\right) \exp (-\gamma t)\right\} .
$$

Let $\beta$ in this case be $\beta_{1}$ to be $\sqrt{\frac{\gamma}{2}}$, then when we choose $\gamma$ large, $\gamma-\beta^{2}$ is large. The inequality becomes

$$
P\left(e_{I-1}^{k+1}\left(X, b_{I-1}, t\right)\right) \exp (-\gamma t) \leq \exp \left(-\beta_{1} S_{I-1}\right) \max _{\bar{D} \times[0, T]}\left\{P\left(e_{I}^{k}\left(X, a_{I}, t\right)\right) \exp (-\gamma t)\right\} .
$$

We deduce

$$
P\left(e_{I-1}^{k+1}\left(X, b_{I-1}, t\right)\right) \exp (-\gamma t) \leq \exp \left(-\beta_{1} S_{I-1}\right) E_{k} .
$$

Moreover, on the $(I-1)$ th domain, at the $(k+1)$ th step, $(5.6)$ leads to

$$
\begin{aligned}
& P\left(e_{I-1}^{k+1}\right)(X, z, t) \exp \left(\beta\left(z-a_{I-1}\right)-\gamma t\right) \\
& \leq \max \left\{\max _{\bar{D} \times[0, T]}\left\{P\left(e_{I-1}^{k+1}\right)\left(X, b_{I-1}, t\right) \exp \left(\beta\left(b_{I-1}-a_{I-1}\right)-\gamma t\right)\right\},\right. \\
& \left.\max _{\bar{D} \times[0, T]}\left\{P\left(e_{I-1}^{k+1}\right)\left(X, a_{I-1}, t\right) \exp (-\gamma t)\right\}\right\} .
\end{aligned}
$$

Since $e_{I-1}^{k+1}\left(X, b_{I-2}, t\right)=e_{I-2}^{k+2}\left(X, b_{I-2}, t\right)$,

$$
\begin{aligned}
& P\left(e_{I-2}^{k+2}\right)\left(X, b_{I-2}, t\right) \exp \left(\beta\left(b_{I-2}-a_{I-1}\right)-\gamma t\right) \\
\leq & \max \left\{\max _{\bar{D} \times[0, T]}\left\{P\left(e_{I-1}^{k+1}\right)\left(X, b_{I-1}, t\right) \exp \left(\beta\left(b_{I-1}-a_{I-1}\right)-\gamma t\right)\right\},\right. \\
& \left.\max _{\bar{D} \times[0, T]}\left\{P\left(e_{I-1}^{k+1}\right)\left(X, a_{I-1}, t\right) \exp (-\gamma t)\right\}\right\} \\
= & \max \left\{\max _{\bar{D} \times[0, T]}\left\{P\left(e_{I-1}^{k+1}\right)\left(X, b_{I-1}, t\right) \exp \left(\beta L_{I-1}-\gamma t\right)\right\},\right. \\
& \left.\max _{\bar{D} \times[0, T]}\left\{P\left(e_{I-1}^{k+1}\right)\left(X, a_{I-1}, t\right) \exp (-\gamma t)\right\}\right\} .
\end{aligned}
$$


Hence

$$
\begin{aligned}
& P\left(e_{I-2}^{k+2}\right)\left(X, b_{I-2}, t\right) \exp \left(\beta S_{I-2}-\gamma t\right) \leq \max \left\{P\left(e_{I-1}^{k+1}\right)\left(X, b_{I-1}, t\right)\right. \\
& \left.\times \exp \left(\beta L_{I-1}-\gamma t\right), P\left(e_{I-1}^{k+1}\right)\left(X, a_{I-1}, t\right) \exp (-\gamma t)\right\}
\end{aligned}
$$

Since

$$
P\left(e_{I-1}^{k+1}\right)\left(X, a_{I-1}, t\right) \exp (-\gamma t) \leq E_{k+1},
$$

(5.7) implies

$$
P\left(e_{I-2}^{k+2}\right)\left(X, b_{I-2}, t\right) \exp \left(\beta S_{I-2}-\gamma t\right) \leq \max \left\{E_{k} \exp \left(\beta L_{I-1}-\beta_{1} S_{I-1}\right), E_{k+1}\right\} .
$$

Thus

$$
P\left(e_{I-2}^{k+2}\right)\left(X, b_{I-2}, t\right) \exp (-\gamma t) \leq \max \left\{E_{k} \exp \left(\beta\left(L_{I-1}-S_{I-2}\right)-\beta_{1} S_{I-1}\right), E_{k+1} \exp \left(-\beta S_{I-2}\right)\right\} .
$$

We choose $\beta=\beta_{2}=\beta_{1} \frac{S_{I-1}}{L_{I-1}}$ such that $\beta_{2}\left(-L_{I-1}+S_{I-2}\right)+\beta_{1} S_{I-1}=\beta_{2} S_{I-2}$; then

$$
P\left(e_{I-2}^{k+2}\right)\left(X, b_{I-2}, t\right) \exp (-\gamma t) \leq \max \left\{E_{k}, E_{k+1}\right\} \exp \left(-\beta_{2} S_{I-2}\right) .
$$

Using the same techniques as the ones that we use to achive (5.7) and (5.8), we can prove that

$$
P\left(e_{I-j}^{k+j}\right)\left(X, b_{I-j}, t\right) \exp (-\gamma t) \leq \max \left\{E_{k}, \ldots, E_{k+j-1}\right\} \exp \left(-\beta_{j} S_{I-j}\right),
$$

where $\beta_{j}=\beta_{1} \frac{S_{I-1}}{L_{I-1}} \ldots \frac{S_{I-j+1}}{L_{I-j+1}}, j=\{2, \ldots, I-1\}$.

Step 2.2. Estimate of the left boundaries of the sub-domains

Consider the first domain, at the $k$ th step, (5.4) infers

$$
P\left(e_{1}^{k}(X, z, t)\right) \exp (\beta z-\gamma t) \leq \max _{\bar{D} \times[0, T]}\left\{P\left(e_{1}^{k}\left(X, b_{1}, t\right)\right) \exp \left(\beta b_{1}-\gamma t\right)\right\} .
$$

Replace $z$ by $a_{2}$, we get

$$
P\left(e_{1}^{k}\left(X, a_{2}, t\right)\right) \exp (-\gamma t) \leq \max _{\bar{D} \times[0, T]}\left\{P\left(e_{1}^{k}\left(X, b_{1}, t\right)\right) \exp (-\gamma t)\right\} \exp \left(\beta\left(-a_{2}+b_{1}\right)\right) .
$$

Since $e_{1}^{k}\left(X, a_{2}, t\right)=e_{2}^{k+1}\left(X, a_{2}, t\right)$,

$$
P\left(e_{2}^{k+1}\left(X, a_{2}, t\right)\right) \exp (-\gamma t) \leq \max _{\bar{D} \times[0, T]}\left\{P\left(e_{1}^{k}\left(X, b_{1}, t\right)\right) \exp (-\gamma t)\right\} \exp \left(\beta S_{1}\right) .
$$

Let $\beta=-\beta_{1}^{\prime}=-\sqrt{\frac{\gamma}{2}}$, then when we choose $\gamma$ large, $\gamma-\beta^{2}$ is large. The inequality becomes

$$
P\left(e_{2}^{k+1}\left(X, a_{2}, t\right)\right) \exp (-\gamma t) \leq \exp \left(-\beta_{1}^{\prime} S_{1}\right) \max _{\bar{D} \times[0, T]}\left\{P\left(e_{1}^{k}\left(X, b_{1}, t\right)\right) \exp (-\gamma t)\right\} .
$$

We deduce

$$
P\left(e_{2}^{k+1}\left(X, a_{2}, t\right)\right) \exp (-\gamma t) \leq \exp \left(-\beta_{1}^{\prime} S_{1}\right) E_{k} .
$$

Moreover, on the second domain, at the $(k+1)$ th step, (5.6) leads to

$$
\begin{aligned}
& P\left(e_{2}^{k+1}\right)(X, z, t) \exp \left(\beta\left(z-a_{2}\right)-\gamma t\right) \\
& \leq \max _{\bar{D} \times[0, T]}\left\{\max _{\bar{D}}\left\{P\left(e_{2}^{k+1}\right)\left(X, b_{2}, t\right) \exp \left(\beta\left(b_{2}-a_{2}\right)-\gamma t\right)\right\},\right. \\
& \left.\max _{\bar{D} \times[0, T]}\left\{P\left(e_{2}^{k+1}\right)\left(X, a_{2}, t\right) \exp (-\gamma t)\right\}\right\} .
\end{aligned}
$$


Since $e_{2}^{k+1}\left(X, a_{3}, t\right)=e_{3}^{k+2}\left(X, a_{3}, t\right)$

$$
\begin{aligned}
& P\left(e_{3}^{k+2}\right)\left(X, a_{3}, t\right) \exp \left(\beta\left(a_{3}-a_{2}\right)-\gamma t\right) \\
\leq & \max \left\{\max _{\bar{D} \times[0, T]}\left\{P\left(e_{2}^{k+1}\right)\left(X, b_{2}, t\right) \exp \left(\beta\left(b_{2}-a_{2}\right)-\gamma t\right)\right\},\right. \\
& \left.\max _{\bar{D} \times[0, T]}\left\{P\left(e_{2}^{k+1}\right)\left(X, a_{2}, t\right) \exp (-\gamma t)\right\}\right\} \\
= & \max \left\{\max _{\bar{D} \times[0, T]}\left\{P\left(e_{2}^{k+1}\right)\left(X, b_{2}, t\right) \exp \left(\beta L_{2}-\gamma t\right)\right\},\right. \\
& \left.\max _{\bar{D} \times[0, T]}\left\{P\left(e_{2}^{k+1}\right)\left(X, a_{2}, t\right) \exp (-\gamma t)\right\}\right\} .
\end{aligned}
$$

Hence

$$
\begin{gathered}
P\left(e_{3}^{k+2}\right)\left(X, a_{3}, t\right) \exp \left(\beta\left(L_{2}-S_{2}\right)-\gamma t\right) \leq \max \left\{P\left(e_{2}^{k+1}\right)\left(X, b_{2}, t\right)\right. \\
\left.\times \exp \left(\beta L_{2}-\gamma t\right), P\left(e_{2}^{k+1}\right)\left(X, a_{2}, t\right) \exp (-\gamma t)\right\}
\end{gathered}
$$

Since

$$
P\left(e_{2}^{k+1}\right)\left(X, b_{2}, t\right) \exp (-\gamma t) \leq E_{k+1},
$$

(5.7) implies

$$
P\left(e_{3}^{k+2}\right)\left(X, a_{3}, t\right) \exp \left(\beta\left(L_{2}-S_{2}\right)-\gamma t\right) \leq \max \left\{E_{k} \exp \left(-\beta_{1}^{\prime} S_{1}\right), E_{k+1} \exp \left(\beta L_{2}\right)\right\}
$$

Thus

$$
P\left(e_{3}^{k+2}\right)\left(X, a_{3}, t\right) \exp (-\gamma t) \leq \max \left\{E_{k} \exp \left(-\beta\left(L_{2}-S_{2}\right)-\beta_{1}^{\prime} S_{1}\right), E_{k+1} \exp \left(\beta S_{2}\right)\right\}
$$

We choose $\beta=-\beta_{2}^{\prime}=-\beta_{1}^{\prime} \frac{S_{1}}{L_{2}}$ such that $-\beta\left(L_{2}-S_{2}\right)-\beta_{1}^{\prime} S_{1}=\beta S_{2}$. Then, we have that

$$
P\left(e_{3}^{k+2}\right)\left(X, a_{3}, t\right) \exp (-\gamma t) \leq \max \left\{E_{k}, E_{k+1}\right\} \exp \left(-\beta_{2}^{\prime} S_{2}\right)
$$

Using the same techniques as the ones that we use to achive (5.10) and (5.11), we can prove that

$$
P\left(e_{j}^{k+j-1}\right)\left(X, a_{j}, t\right) \exp (-\gamma t) \leq \max \left\{E_{k}, \ldots, E_{k+j-2}\right\} \exp \left(-\beta_{j-1}^{\prime} S_{j-1}\right)
$$

where $\beta_{j}^{\prime}=\beta_{1}^{\prime} \frac{S_{1}}{L_{2}} \ldots \frac{S_{j-1}}{L_{j}}, j=\{2, \ldots, I-1\}$. 
Step 2.3. Convergence result

From the fact that $\bar{\epsilon}=\sqrt{\frac{\gamma}{2}} \frac{S_{1} \ldots S_{I-1}}{L_{2} \ldots L_{I-1}}$, and

$$
\bar{E}_{k}=\max _{j \in[0, I-1]}\left\{E_{k+j}\right\},
$$

(5.9) and (5.12) give us

$$
\bar{E}_{k+1} \leq \bar{E}_{k} \exp (-\bar{\epsilon}), \forall k \in \mathbb{N},
$$

or

$$
\bar{E}_{n} \leq \bar{E}_{0} \exp (-n \bar{\epsilon}), \forall n \in \mathbb{N}
$$

Hence $E_{k}$ tends to 0 as $k$ tends to infinity, which gives

$$
\lim _{k \rightarrow \infty} \max _{j=\overline{1, I}}\left\|P\left(e_{j}^{k}\right)\right\|_{C\left(\overline{\Omega_{j} \times(0, T)}\right)}=0,
$$

and we get the linear convergence of the algorithm.

Acknowledgements. The author would like to thank Professor Martin Gander, Professor Laurence Halpern and Professor Enrique Zuazua for their help.

\section{REFERENCES}

[1] J.-D. Benamou and B. Desprès, A domain decomposition method for the Helmholtz equation and related optimal control problems. J. Comput. Phys. 136 (1997) 68-82.

[2] K. Burrage, C. Dyke and B. Pohl, On the performance of parallel waveform relaxations for differential systems. Appl. Numer. Math. 20 (1996) 39-55.

[3] Th. Cazenave and A. Harau, An introduction to semilinear evolution equations, vol. 13 of Oxford Lect. Ser. Math. Applic. The Clarendon Press Oxford University Press, New York (1998). Translated from the 1990 French original by Yvan Martel and revised by the authors.

[4] E.B. Davies, Heat kernels and spectral theory, in vol. 92 of Cambridge Tracts in Mathematics. Cambridge University Press, Cambridge (1989).

[5] S. Descombes, V. Dolean and M.J. Gander, Schwarz waveform relaxation methods for systems of semi-linear reaction-diffusion equations, in Domain Decomposition Methods (2009).

[6] L.C. Evans, Partial differential equations, Graduate Studies in Mathematics, in vol. 19 of Amer. Math. Soc. Providence, RI (1998).

[7] A. Friedman, Partial differential equations of parabolic type. Prentice-Hall Inc., Englewood Cliffs, N.J (1964).

[8] M.J. Gander and L. Halpern. Optimized Schwarz waveform relaxation methods for advection reaction diffusion problems. SIAM J. Numer. Anal. 45 (2007) 666-697.

[9] M.J. Gander, L. Halpern and F. Nataf, Optimal convergence for overlapping and non-overlapping Schwarz waveform relaxation, in Eleventh International Conference on Domain Decomposition Methods (London, 1998). DDM.org, Augsburg (1999) 27-36.

[10] M.J. Gander, A waveform relaxation algorithm with overlapping splitting for reaction diffusion equations. Numer. Linear Algebra Appl. 6 (1999) 125-145. Czech-US Workshop in Iterative Methods and Parallel Computing, Part 2 (Milovy 1997).

[11] M.J. Gander, L. Halpern and F. Nataf, Optimized Schwarz methods. In Domain decomposition methods in sciences and engineering (Chiba, 1999). DDM.org, Augsburg (2001) 15-27.

[12] M.J. Gander and A.M. Stuart, Space time continuous analysis of waveform relaxation for the heat equation. SIAM J. 19 (1998) 2014-2031.

[13] M.J. Gander and H. Zhao, Overlapping Schwarz waveform relaxation for the heat equation in $n$ dimensions. BIT 42 (2002) 779-795.

[14] E. Giladi and H.B. Keller, Space-time domain decomposition for parabolic problems. Numer. Math. 93 (2002) $279-313$.

[15] G.M. Lieberman. Second order parabolic differential equations. World Scientific Publishing Co. Inc., River Edge, NJ (1996).

[16] P.-L. Lions, On the Schwarz alternating method I. In First International Symposium on Domain Decomposition Methods for Partial Differential Equations (Paris, 1987). SIAM, Philadelphia, PA (1988) 1-42.

[17] P.-L. Lions, On the Schwarz alternating method II. Stochastic interpretation and order properties, in Domain decomposition methods (Los Angeles, CA, 1988). SIAM, Philadelphia, PA (1989) 47-70. 
[18] P.-L. Lions, On the Schwarz alternating method III. A variant for nonoverlapping subdomains, in Third International Symposium on Domain Decomposition Methods for Partial Differential Equations (Houston, TX 1989). SIAM, Philadelphia, PA (1990) 202-223.

[19] S.H. Lui, On Schwarz methods for monotone elliptic PDEs, in Domain decomposition methods in sciences and engineering (Chiba, 1999). DDM.org, Augsburg (2001) 55-62.

[20] S.-H. Lui, On monotone iteration and Schwarz methods for nonlinear parabolic PDEs. J. Comput. Appl. Math. 161 (2003) 449-468.

[21] P. Quittner and Ph. Souplet, Superlinear parabolic problems, Blow-up, global existence and steady states. Birkhäuser Advanced Texts: Basler Lehrbücher. [Birkhäuser Advanced Texts: Basel Textbooks]. Birkhäuser Verlag, Basel (2007).

[22] M.-B. Tran, Parallel Schwarz waveform relaxation method for a semilinear heat equation in a cylindrical domain. C. R. Math. Acad. Sci. Paris 348 (2010) 795-799. 